Comment. Math. Helv. 73 (1998) 259-297

(C) 1998 Birkhäuser Verlag, Basel

0010-2571/98/020259-39\$1.50+0.20/0

Commentarii Mathematici Helvetici

\title{
The structure of branching in Anosov flows of 3-manifolds
}

Sérgio R. Fenley*

\begin{abstract}
In this article we study the topology of Anosov flows in 3-manifolds. Specifically we consider the lifts to the universal cover of the stable and unstable foliations and analyze the leaf spaces of these foliations. We completely determine the structure of the non Hausdorff points in these leaf spaces. There are many consequences: (1) when the leaf spaces are non Hausdorff, there are closed orbits in the manifold which are freely homotopic, (2) suspension Anosov flows are, up to topological conjugacy, the only Anosov flows without free homotopies between closed orbits, (3) when there are infinitely many stable leaves (in the universal cover) which are non separated from each other, then we produce a torus in the manifold which is transverse to the Anosov flow and therefore is incompressible, (4) we produce non Hausdorff examples in hyperbolic manifolds and derive important properties of the limit sets of the stable/unstable leaves in the universal cover.
\end{abstract}

Mathematics Subject Classification (1991). Primary: 58F25, 58F18, 58F15, 57R30; Secondary: 57M10, 57M99, 58F22.

Keywords. Anosov flows, stable and unstable foliations, closed orbits, homotopic behavior of closed orbits, transversal submanifolds, non Hausdorff manifolds, hyperbolic manifolds and limit sets.

\section{Introduction}

In this article we study the topological structure of the lifts to the universal cover of the stable and unstable foliations of 3-dimensional Anosov flows. In particular we consider the case when these foliations do not have Hausdorff leaf space. We completely determine the structure of the set of non separated leaves from a given leaf in one of these foliations. We show that these leaves project to leaves in the manifold containing periodic orbits of the flow and produce a non trivial free homotopy between closed orbits of the flow. As a consequence suspensions are characterized, up to topological conjugacy, as the only 3-dimensional Anosov flows without freely homotopic closed orbits. Furthermore we establish a connec-

* Research partially supported by NSF grant DMS-9201744 and an NSF Mathematical Sciences Postdoctoral Fellowship. 
tion with the topology of the manifold: if there are infinitely many leaves not separated from each other then there is an incompressible torus transverse to the flow. Transitivity is not assumed for these results. Finally we specialize to Anosov flows in hyperbolic 3-manifolds: we produce many non Hausdorff examples and then derive some important properties of the limit sets of leaves in the universal cover.

The study of the topological structure of the lifted foliations of an Anosov flow in a closed manifold was introduced in a remarkable paper of Verjovsky [Ve] in order to study codimension one Anosov flows. If the lifted stable foliation has Hausdorff leaf space, then the leaf space is homeomorphic to the set of real numbers and we say that the stable foliation in the manifold is $\mathbf{R}$-covered. An important fact for 3-dimensional Anosov flows is that the stable foliation is $\mathbf{R}$-covered if and only if the unstable foliation is also $\mathbf{R}$-covered [Fe3,Ba2], in which case the flow is said to be $\mathbf{R}$-covered. In this article we restrict to Anosov flows in (closed) 3-manifolds.

Two early uses of this technique were: (1) Ghys [Gh] showed that an Anosov flow in a Seifert fibered space is $\mathbf{R}$-covered. This was an essential step in showing that the flow is, up to finite covers, topologically conjugate to the geodesic flow in the unit tangent bundle of a closed surface of negative curvature (briefly, a geodesic flow). (2) If the fundamental group of the manifold is solvable then the $\mathbf{R}$-covered property, proved by Barbot [Ba1,Ba2], is again an essential step in Plante's proof $[\mathrm{Pl} 2, \mathrm{Pl} 3]$ that the flow is topologically conjugate to the suspension of an Anosov diffeomorphism of the torus (a suspension). In fact this last result holds for any codimension one Anosov flow. This highlights the importance of the topology of the lifted foliations in order to understand the flow.

More recently, a lot of information has been gained by analysing not just the individual leaf spaces, but rather the joint topological structure of the stable and unstable foliations. Using this and Dehn surgery on closed orbits of suspensions or geodesic flows [Fr,Go], a large family of examples was constructed where every closed orbit of the flow is freely homotopic to infinitely many other closed orbits [Fe3]. This never happens for suspensions or geodesic flows, and was thought to be impossible for any Anosov flow.

Our initial motivation was to understand Anosov flows in hyperbolic 3-manifolds, of which there are many examples [Go, Ch], but which are still fairly misterious. Up to now, the only technique that yields any information when the manifold is hyperbolic is the topological theory mentioned above. For instance the topological theory gives information about metric properties of flow lines: a flow is said to be quasigeodesic if flow lines are uniformly efficient (up to a bounded multiplicative distortion) in measuring distances in relative homotopy classes. Suspensions and geodesic flows are always quasigeodesic and there are many quasigeodesic "pseudo-Anosov" flows in hyperbolic 3-manifolds [Ca-Th,Mos1,Mos2,Fe-Mo] (a pseudo-Anosov flow is a generalization of an Anosov flow, where one allows finitely many singular orbits, which have prong type singularities, see [Fe-Mo]). The quasi- 
geodesic property is extremely important in hyperbolic 3-manifolds [Th1,Mor] and was used for instance in the proof of the geometrization conjecture for Haken manifolds. Using the topological theory and the Dehn surgery construction mentioned above, one produces a large family of Anosov flows in hyperbolic manifolds which are not quasigeodesic [Fe3]. All these examples are R-covered Anosov flows. One important question is to understand how such flows interact with the hyperbolic structure and the ideal compactification of the universal cover. We will return to this question later.

Barbot [Ba3,Ba4] also used this topological theory to study Anosov flows and proved the following remarkable result: Assume that there is a Seifert fibered piece in the torus decomposition of the manifold [Jo,Ja-Sh] and suppose that the corresponding fiber is not freely homotopic to a closed orbit of the flow. First isotopically adjust the boundary tori of the Seifert fibered piece to be as transverse to the flow as possible [Ba3]. It follows that the flow restricted to that piece is topologically conjugate to a (generalized) geodesic flow on the unit tangent bundle of a compact surface with boundary. If the manifold is a graph manifold (that is, all pieces of torus decomposition are Seifert fibered) and all fibers satisfy the condition above, then the flow in $M$ is, up to topological conjugay, obtained by Dehn surgery on finitely many closed orbits of a geodesic flow [Ba4]. Using this Barbot [Ba4] has obtained the first known examples of graph manifolds which are neither torus bundles over the circle, nor Seifert fibered and which do not admit Anosov flows.

The results above are in great part due to a complete characterization of the possible joint topological structures of $\mathbf{R}$-covered Anosov flows [Fe3,Ba2]. On the other hand very little is known about the non $\mathbf{R}$-covered case, for the simple reason that their structure is not understood at all.

It is easy to show that intransitivity implies that the flow is not $\mathbf{R}$-covered [So,Ba1] and for many years there was a great effort in trying to prove that these two properties are equivalent [Ve, $\mathrm{Gh}, \mathrm{Fe} 3, \mathrm{Ba} 2]$. However in a surprising development Bonatti and Langevin [Bo-La] have recently constructed a transitive, non R-covered Anosov flow in dimension 3. Subsequently Brunella $[\mathrm{Br}]$ produced a large class of examples by doing Dehn surgery on geodesic flows. The common tool used to show that the flows are not $\mathbf{R}$-covered is the existence of an embedded torus transverse to the flow, which is then incompressible. Hence the underlying manifolds are not hyperbolic.

One fundamental question which remained to be answered was whether the manifold $M$ being hyperbolic would imply that any Anosov flow in $M$ has to be R-covered. A positive answer would have enormous consequences: no Anosov flow in a hyperbolic 3-manifold could be quasigeodesic [Fe3] and in such flows every closed orbit would be freely homotopic to infinitely many other closed orbits [Fe3]. In this article we answer this question in the negative:

Theorem A. There is a large class of transitive, non $\mathbf{R}$-covered Anosov flows 
where the underlying 3-manifold is hyperbolic. This includes all Anosov flows in non orientable, hyperbolic 3-manifolds.

Therefore transitive non $\mathbf{R}$-covered Anosov flows can occur in graph manifolds [Bo-La], in hyperbolic 3-manifolds and in manifolds containing Seifert fibered and hyperbolic pieces in their torus decomposition [Br]. This highlights the importance of understanding the structure of non $\mathbf{R}$-covered Anosov flows, which up to now are completely mysterious. The main goal of this article is to start a systematic study of Anosov flows which are not $\mathbf{R}$-covered, where we then say the lifted foliations have branching. We will not a priori assume that the manifold is hyperbolic or that the flow is transitive.

This leads us to two basic and very important questions: (1) when can branching occur and (2) what are the possible structures of branching in Anosov flows of 3-manifolds? In this article we address question (2) and completely determine the local structure of branching. We then show that the branching structure is strongly related to the dynamics of the flow, the topology of the manifold and the metric behavior of the stable and unstable foliations.

Let then $\Phi$ be an Anosov flow in $M^{3}$ with two dimensional stable and unstable foliations $\mathcal{F}^{s}, \mathcal{F}^{u}$. Here $M$ is always closed. Let $\widetilde{\mathcal{F}}^{s}, \widetilde{\mathcal{F}}^{u}$ be the respective lifts

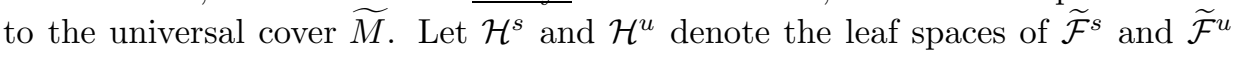
respectively. If $\mathcal{F}^{s}$ is not $\mathbf{R}$-covered, $\mathcal{H}^{s}$ is not Hausdorff, and the branching leaves of $\widetilde{\mathcal{F}}^{s}$ correspond to the non Hausdorff points in $\mathcal{H}^{s}$. Two leaves $F \neq F^{\prime}$ of $\widetilde{\mathcal{F}}^{s}$ form a branching pair if the corresponding points in $\mathcal{H}^{s}$ are not separated from each other. Equivalently $F, F^{\prime}$ do not have disjoint saturated neighborhoods in $\widetilde{M}$, where a saturated neighborhood of $\widetilde{\mathcal{F}}^{s}$ is an open set which is a union of leaves of $\widetilde{\mathcal{F}}^{s}$.

Since $\widetilde{M}$ is simply connected, $\widetilde{\mathcal{F}}^{s}$ and $\widetilde{\mathcal{F}}^{u}$ are always transversely orientable and an orientation is fixed. Then there is a notion of branching in the positive or negative directions. The first important result was proved in [Fe5]: Suppose that $\Phi$ is transitive. If there is branching in the positive direction of (say) the stable foliation then this foliation also has branching in the negative direction. This concerns the "global" structure of branching.

Here we analyse the "local" structure of branching. In general the local structure of branching in the lifted foliations can be very complicated [Im]. We show that branching in Anosov foliations is of a simple type which is very rigid. For simplicity the theorems are stated for $\widetilde{\mathcal{F}}^{s}$ but work equally well for $\widetilde{\mathcal{F}}^{u}$.

A leaf of $\widetilde{\mathcal{F}}^{s}$ or $\widetilde{\mathcal{F}}^{u}$ is said to be periodic if it is left invariant by a non trivial covering translation of the universal cover. Equivalently, its image in $M$ contains a closed orbit of $\Phi$.

Theorem B. Let $\Phi$ be an Anosov flow in $M^{3}$. If $F$ is a branching leaf of $\widetilde{\mathcal{F}}^{s}$, then $F$ is periodic. 


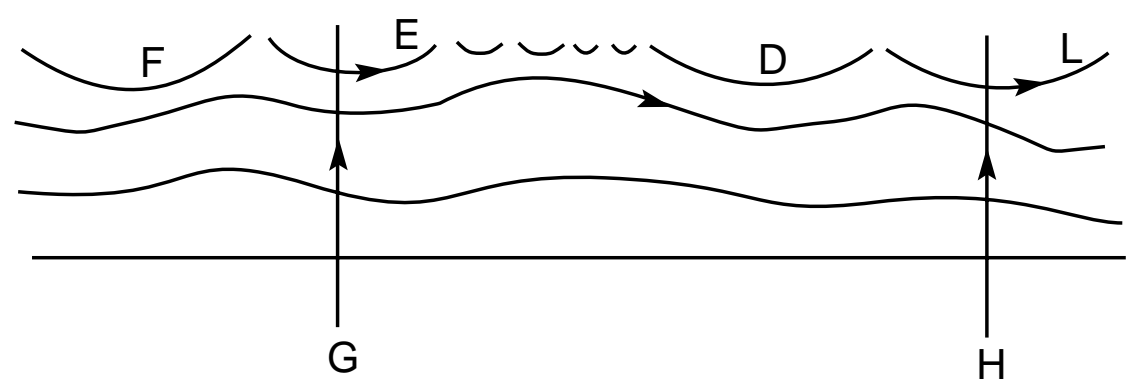

Figure 1. The set of non separated leaves from $F \in \widetilde{\mathcal{F}}^{s}$ and in the back of $F$. $D$ is between $E$ and $L$.

Theorem B should be interpreted as a rigidity result in the sense that periodic leaves are "rigid", while non periodic leaves are non rigid. This is best seen in the manifold $M$ : if the stable leaf (in $M$ ) is periodic then it contains a closed orbit of $\Phi$ and every orbit in the leaf is forward asymptotic to this closed orbit. The nearby returns (in forward direction) are in the same local stable leaf. In case the leaf is not periodic, then the orbits in the leaf limit in points of $M$, but the nearby returns are always in distinct local stable leaves. This means that when lifted to the universal cover one can slightly perturb the local structure, producing a contradiction.

Our next goal is to understand the local structure of branching. Let $F$ be a leaf of $\widetilde{\mathcal{F}}^{s}$ which is a branching leaf. Let $\mathcal{E}_{b}(F)$ be the set of leaves of $\widetilde{\mathcal{F}}^{s}$ which are non separated from $F$ and are either equal to $F$ or are contained in the back of $F$. Similarly define $\mathcal{E}_{f}(F)$. We show there is a natural order in $\mathcal{E}_{b}(F)$ given by: if $E \neq L \in \mathcal{E}_{b}(F)$ then we say that $E<L$ if there are $G, H \in \widetilde{\mathcal{F}}^{u}$ with $G \cap E \neq \emptyset, H \cap L \neq \emptyset$ and $G$ is in the negative side of $H$, see fig. 1. Using this we can say that a branching leaf $D \in \mathcal{E}_{b}(F)$ is between $E$ and $L$ if $E<D<L$.

One measure of the complexity of branching is the number of branching leaves between any $E, L \in \mathcal{E}_{b}(F)$. A priori there could be infinitely many in between branching leaves producing a very complicated structure. However we prove:

Theorem C. Let $\Phi$ be an Anosov flow in $M^{3}$. Let $F$ be a branching leaf of $\widetilde{\mathcal{F}}^{s}$ and $\mathcal{E}_{b}(F)$ be the set of non separated leaves from $F$ and in the back of $F$ ( $F$ is included in $\mathcal{E}_{b}(F)$ ), with the order defined above. Then either

(1) $\mathcal{E}_{b}(F)$ is finite, hence order isomorphic to $\{1,2, \ldots, n\}$ or,

(2) $\mathcal{E}_{b}(F)$ if infinite and order isomorphic to the set of integers $\mathbf{Z}$.

In particular given any $E, L \in \mathcal{E}_{b}(F)$, there are only finitely many branching leaves between them. Analogous results hold for $\mathcal{E}_{f}(F)$.

Notice that there are examples where both $\mathcal{E}_{b}(F)$ and $\mathcal{E}_{f}(F)$ contain leaves other than $F$. This is what happens in the intransitive examples created by Franks 
and Williams [Fr-Wi]. A careful analysis of those examples shows that for any branching leaf $F \in \widetilde{\mathcal{F}}^{s}$, then both $\mathcal{E}_{b}(F)$ and $\mathcal{E}_{f}(F)$ contain exactly two elements.

As in the case of theorem $B$, there is a rigidity proof of theorem $C$. However the rigidity proof is quite long and complicated. Our tactic will be to first show the following result:

Theorem D. Let $\Phi$ be an Anosov flow in $M^{3}$ and let $(F, L)$ be a branching pair of $\widetilde{\mathcal{F}}^{s}$. Let $g$ be a non trivial covering translation with $g(F)=F$ and so that $g$ preserves transversal orientations to $\widetilde{\mathcal{F}}^{s}, \widetilde{\mathcal{F}}^{u}$. Then $g(L)=L$.

Using the important idea of lozenges (see definition in section 3) and a key result from [Fe4], we show that theorem $C$ follows easily from theorem $D$, except that to rule out the case that $\mathcal{E}_{b}(F)$ is order isomorphic to the natural numbers $\mathbf{N}$ we need theorem $F$ below. Section 4 contains a more detailed description of the set $\mathcal{E}_{b}(F)$.

Theorem D also implies that $\pi(F)$ and $\pi(L)$ contain freely homotopic closed orbits and highlights the pervasiviness of freely homotopic closed orbits. This shows that the topological structure of $\widetilde{\mathcal{F}}^{s}, \widetilde{\mathcal{F}}^{u}$ is intimately connected with the dynamics of the flow:

Corollary E. Let $\Phi$ be an Anosov flow in $M^{3}$. Then $\Phi$ is topologically conjugate to a suspension of an Anosov diffeomorphism of the torus if and only if there are no freely homotopic closed orbits of $\Phi$ (including non trivial free homotopies of a closed orbit to itself).

Corollary $E$ does not assume that $\Phi$ is not $\mathbf{R}$-covered. Another consequence of theorem $D$ is the following:

Theorem F. Let $\Phi$ be a non $\mathbf{R}$-covered Anosov flow in $M^{3}$. Then up to the action of covering translations, there are finitely many branching leaves in $\widetilde{\mathcal{F}}^{s}$. Equivalently there are finitely many distinguished closed orbits of $\Phi$ in $M$ so that their stable leaves lift to branching leaves in the universal cover.

We again stress that these results on the structure of branching are the most general possible, because there is no assumption on the manifold nor on the flow. In particular we do not assume that the flow is transitive. Theorems $B, C, D$ and $F$ were previously proved under the assumption that $M$ has negatively curved fundamental group and furthermore that the flow is quasigeodesic [Fe4]. This last hypothesis is a very strong assumption and made the proofs relatively easy. The techniques used here are different because in general there are no metric properties available to use. The proof only uses the topological structure of $\widetilde{\mathcal{F}}^{s}$.

We also show that the structure of branching is strongly related to the topology of the ambient manifold. We say that there is infinite branching in $\widetilde{\mathcal{F}}^{s}$ if there 
is an infinite collection of leaves of $\widetilde{\mathcal{F}}^{s}$, all of which are non separated from each other. Otherwise we say that $\widetilde{\mathcal{F}}^{s}$ has only finite branching.

Theorem G. Let $\Phi$ be an Anosov flow in $M^{3}$ orientable so that there is infinite branching in $\widetilde{\mathcal{F}}^{s}$. Then there is associated infinite branching in $\widetilde{\mathcal{F}}^{u}$ and there is an embedded torus $T$ transverse to $\Phi$. Hence $T$ is incompressible. In particular $M$ is toroidal and cannot be hyperbolic and neither can $\pi_{1}(M)$ be negatively curved.

A big part of theorem $G$ follows quickly from theorem $D$ and 3-manifold topology, namely the fact that $M$ atoroidal implies there is no infinite branching (corollary 4.8). However this "quick" proof uses 2 deep results: (1) The general torus theorem $[\mathrm{Ga}]$ which in turn depends on the solution of the Seifert fibered conjecture, and (2) The characterization of Anosov flows in Seifert fibered 3-manifolds [Gh]. We give a proof of theorem $G$ which depends only on the topological structure of $\widetilde{\mathcal{F}}^{s}$ and $\widetilde{\mathcal{F}}^{u}$.

We remark that infinite branching does occur, for example in the BonattiLangevin flow. Conversely theorem $A$ yields many examples of non trivial finite branching.

We also describe in detail the structure induced by infinite branching. This uses "product regions" (see section 3), a tool which also has applications in [Fe8].

With the description of branching in general given by theorems $B, C, D$ and $F$, we can then specialize to Anosov flows in hyperbolic 3 -manifolds. In that case $\widetilde{M}$ is compactified with a sphere at infinity $S_{\infty}^{2}$ and it is extremely important to understand the asymptotic behavior of stable and unstable leaves in $\widetilde{M}$ and in particular to study their limit sets [Th1,Th2,Mor,Bon]. The intrinsic geometry of a leaf $F$ of $\widetilde{\mathcal{F}}^{s}$ or $\widetilde{\mathcal{F}}^{u}$ is always negatively curved in the large and there is an intrinsic ideal boundary $\partial_{\infty} F$. We say that $\widetilde{\Phi}$ has the continuous extension property if the embedding $\varphi: F \rightarrow \widetilde{M}$ extends continuously to $\varphi: F \cup \partial_{\infty} F \rightarrow \widetilde{M} \cup S_{\infty}^{2}$, for any leaf $F$ in $\widetilde{\mathcal{F}}^{s}$ or $\widetilde{\mathcal{F}}^{u}$. This property can be defined for any Reebless codimension 1 foliation in hyperbolic manifolds [Fe2] and it is true for fibrations [Ca-Th] and many depth one foliations $[\mathrm{Fe} 1, \mathrm{Fe} 10]$. This property is weaker than quasigeodesic behavior of $\Phi$ [Fe3]. Recall that the limit set of $B$ is the set of accumulation points of $B$ in $S_{\infty}^{2}$. In this article we use the structure of branching to analyse limit sets of leaves in connection with the continuous extension property:

Theorem H. Let $\Phi$ be a non $\mathbf{R}$-covered Anosov flow in $M^{3}$ hyperbolic. Suppose that $\widetilde{\Phi}$ has the continuous extension property. Then the limit set $\Lambda_{F}$ of any leaf $F$ of $\widetilde{\mathcal{F}}^{s}$ or $\widetilde{\mathcal{F}}^{u}$ is a Sierpinski curve, that is, the complement of a countable, dense union of open disks in $S_{\infty}^{2}$. In addition there is $k<2$ so that $\Lambda_{F}$ has Hausdorff dimension $<k$ for any $F \in \widetilde{\mathcal{F}}^{s}$ or $\widetilde{\mathcal{F}}^{u}$, so in particular $\Lambda_{F}$ has zero Lebesgue area.

This result (except for the last statement) also works under the assumption that $\pi_{1}(M)$ is negatively curved. Theorem $H$ is a significant improvement over results 
in [Fe4]: in [Fe4] we assumed the stronger hypothesis that $\Phi$ is quasigeodesic and were only able to show that $\Lambda_{F}$ is neither $S_{\infty}^{2}$ nor a Jordan curve.

In a forthcoming paper [Fe8] we use the results of this article to study Anosov flows in toroidal manifolds, specifically to study incompressible tori in such manifolds. It is of great interest to find, in the isotopy class of the torus, the best position with respect to the flow $[\mathrm{Ba} 3, \mathrm{Ba} 4]$. We prove:

Theorem. ([Fe8]) Let $\Phi$ be an Anosov flow in $M^{3}$ and let $T$ an incompressible torus in $M$. Suppose that no loop in $T$ is freely homotopic to a closed orbit of $\Phi$. Then $\Phi$ is topologically conjugate to a suspension Anosov flow. Furthermore $T$ is isotopic to a torus transverse to $\Phi$.

This article is organized as follows: in the next section we develop background material and in section 3 we prove that branching leaves are periodic (theorem $B)$. Section 4 is the core of the paper, where theorems $D, C, F$, corollary $E$ and part of theorem $G$ are proved. Section 5 studies product regions and applies this to completely describe infinite branching. For the sake of presentation we collect the results concerning hyperbolic manifolds in the last two sections: In section 6 we produce non $\mathbf{R}$-covered Anosov flows in hyperbolic 3-manifolds and in the final section we study the continuous extension property.

We thank Bill Thurston for encouragement and many helpful conversations relating to this work. We also thank Thierry Barbot for useful suggestions to a first version of this article. Finally we thank the referee for an extremely careful reading of the manuscript and for inumerous suggestions which greatly improved the paper and also simplified some of the proofs.

\section{Background}

Let $\Phi_{t}: M^{3} \rightarrow M^{3}$ be a nonsingular flow in a closed, connected Riemannian manifold $M$. The flow $\Phi$ is Anosov if there is a continuous decomposition of the tangent bundle $T M$ as a Whitney sum $T M=E^{0} \oplus E^{s} \oplus E^{u}$ of continuous $D \Phi_{t}$ invariant subbundles and there are constants $\mu_{0} \geq 1, \mu_{1}>0$ so that:

(i) $E^{0}$ is one dimensional and tangent to the flow,

(ii) $\left\|D \Phi_{t}(v)\right\| \leq \mu_{0} e^{-\mu_{1} t}\|v\|$ for any $v \in E^{s}, t \geq 0$,

(iii) $\left\|D \Phi_{-t}(v)\right\| \leq \mu_{0} e^{-\mu_{1} t}\|v\|$ for any $v \in E^{u}, t \geq 0$.

The bundles $E^{s}, E^{u}$ integrate to one dimensional foliations $\mathcal{F}^{s s}, \mathcal{F}^{u u}$ : the strong stable and strong unstable foliations of the flow [An]. The bundles $E^{0} \oplus E^{s}$ and $E^{0} \oplus E^{u}$ are also integrable [An] producing 2-dimensional foliations $\mathcal{F}^{s}, \mathcal{F}^{u}$ which are the stable and unstable foliations of the flow.

The flow is said to be orientable if both $\mathcal{F}^{s}, \mathcal{F}^{u}$ are transversely orientable. There is always a regular cover of order $\leq 4$ where the lifted $\mathcal{F}^{s}$ and $\mathcal{F}^{u}$ are transversely orientable. Whenever possible we lift to such a cover. 
The leaves of $\mathcal{F}^{s}, \mathcal{F}^{u}$ are either topological planes, annuli or Möebius bands. The last two correspond exactly to leaves containing closed orbits of $\Phi$. There is at most one closed orbit in a leaf of $\mathcal{F}^{s}$, in which case all other orbits are forward asymptotic to it. Similarly for $\mathcal{F}^{u}$.

The foliation $\mathcal{F}^{s}$ is Reebless, so Novikov's theorem [No] implies that given any closed orbit $\gamma$ of $\Phi, \gamma^{n}$ is not null homotopic for any $n \neq 0$.

Let $\pi: \widetilde{M} \rightarrow M$ be the universal covering space of $M$. This notation will be fixed throughout the article. The Anosov foliations $\mathcal{F}^{s}, \mathcal{F}^{u}$ lift to foliations $\widetilde{\mathcal{F}}^{s}, \widetilde{\mathcal{F}}^{u}$ in $\widetilde{M}$. The leaves of $\widetilde{\mathcal{F}}^{s}, \widetilde{\mathcal{F}}^{u}$ are topological planes, so $\widetilde{M}$ is homeomorphic to $\mathbf{R}^{3}$ $[\mathrm{Pa}]$. Therefore $M$ is irreducible that is every embedded sphere in $M$ bounds a 3-ball. The induced flow in $\widetilde{M}$ is denoted by $\widetilde{\Phi}$.

Let $\mathcal{O}$ be the orbit space of $\widetilde{\Phi}$ obtained by collapsing flow lines to points and let $\Theta: \widetilde{M} \rightarrow \mathcal{O}$ be the projection map. A fundamental result which will be used throughout this article is that $\mathcal{O}$ is Hausdorff and hence homeomorphic to $\mathbf{R}^{2}$ [Fe3]. This is a significant simplification because now much of the analysis can be done in dimension 2 instead of dimension 3 . We stress that $\mathcal{O}$ is only a topological object - there is no natural metric in $\mathcal{O}$ since the flow direction contracts and expands distances in $\widetilde{M}$. Therefore arguments that involve distances have to be done in $\widetilde{M}$, while topological arguments (e.g. leaves $F_{i}$ converge to $F$, or leaf $F$ intersects leaf $G$ ) can be done in $\mathcal{O}$.

The foliations $\widetilde{\mathcal{F}}^{s}, \widetilde{\mathcal{F}}^{u}$ induce two transverse 1-dimensional foliations in $\mathcal{O}$, which will also be denoted by $\widetilde{\mathcal{F}}^{s}, \widetilde{\mathcal{F}}^{u}$. By an abuse of notation we will many times identify sets in $\widetilde{M}$ or orbits of $\widetilde{\Phi}$ to their respective images in $\mathcal{O}$.

The fundamental group $\pi_{1}(M)$ is isomorphic to the set of covering translations of $\widetilde{M}$. We will usually assume one such identification is fixed. Given a covering translation $g$, we will also denote by $g$ its action on $\mathcal{H}^{s}, \mathcal{H}^{u}$ (the leaves spaces of $\left.\widetilde{\mathcal{F}}^{s}, \widetilde{\mathcal{F}}^{u}\right)$.

Let $W^{s}(x)$ be the leaf of $\mathcal{F}^{s}$ containing $x$ and similarly define $W^{u}(x), W^{s s}(x)$, $W^{u u}(x), \widetilde{W}^{s}(x), \widetilde{W}^{u}(x), \widetilde{W}^{s s}(x)$ and $\widetilde{W}^{u u}(x)$. In the same way if $\alpha$ is an orbit of $\Phi$ we define $W^{s}(\alpha)$, etc.. General references for Anosov flows are [An], [An-Si], [Bow], [Sh] and [Sm].

An incompressible surface $\left(\neq \mathbf{S}^{2}\right)$ is an embedded surface in $M^{3}$ which is injective in the fundamental group level. A manifold is toroidal if it contains an incompressible torus and atoroidal otherwise.

\section{Periodic branching leaves}

In this section we prove theorem $\mathrm{B}$ of the introduction. The following definitions will be useful. If $L$ is a leaf of $\widetilde{\mathcal{F}}^{s}$ or $\widetilde{\mathcal{F}}^{u}$, then a half leaf of $L$ is a connected component $A$ of $L-\gamma$, where $\gamma$ is any full orbit in $L$. The closed half leaf is $\bar{A}=A \cup \gamma$ and its boundary is $\partial A=\gamma$. A flow band $B$ defined by orbits $\alpha \neq \beta$ in $L$ is the connected component of $L-\{\alpha, \beta\}$ which is not a half leaf of $L$. The closed 
flow band associated to it is $\bar{B}=B \cup\{\alpha, \beta\}$ and its boundary is $\partial B=\{\alpha, \beta\}$.

Since $\widetilde{M}$ is simply connected, $\widetilde{\mathcal{F}}^{s}$ and $\widetilde{\mathcal{F}}^{u}$ are transversely orientable. Choose one such orientation. Notice that in general, covering translations may not preserve transversal orientations.

For $p \in \widetilde{M}$, let $\widetilde{W}_{+}^{s}(p)$ be the half leaf of $\widetilde{W}^{s}(p)$ defined by $\widetilde{\Phi}_{\mathbf{R}}(p)$ and the positive transversal orientation to $\widetilde{\mathcal{F}}^{u}$ at $p$. It is called a positive half leaf of $W^{s}(p)$. Similarly define $\widetilde{W}_{-}^{s}(p)$ (a negative half leaf), and also define $\widetilde{W}_{+}^{u}(p)$ and $\widetilde{W_{-}^{u}}(p)$.

A fundamental fact for us is that any leaf $L$ in $\widetilde{\mathcal{F}}^{s}$ or $\widetilde{\mathcal{F}}^{u}$ separates $\widetilde{M}$. This is a consequence of $\widetilde{M}$ being simply connected and $\widetilde{\mathcal{F}}^{s}, \widetilde{\mathcal{F}}^{u}$ being Reebless, which together imply that $L$ is properly embedded [No]. The front of $L$ is the component of $\widetilde{M}-L$ defined by the positive transversal orientation to $L$ We also call this the positive side of $L$. Similarly define the back (or negative side) of $L$. For $p \in \widetilde{M}$ let $\widetilde{W}_{+}^{s s}(p)=\widetilde{W}_{+}^{s}(p) \cap \widetilde{W}^{s s}(p)$. Similarly define $\widetilde{W}_{-}^{s s}(p), \widetilde{W}_{+}^{u u}(p)$ and $\widetilde{W}_{-}^{u u}(p)$.

If $F \in \widetilde{\mathcal{F}}^{s}$ and $G \in \widetilde{\mathcal{F}}^{u}$ then $F$ and $G$ intersect in at most one orbit, since two intersections would force a tangency of $\widetilde{\mathcal{F}}^{s}$ and $\widetilde{\mathcal{F}}^{u}$. This is easiest seen in $\mathcal{O}$, as $\widetilde{\mathcal{F}}^{s}$ and $\widetilde{\mathcal{F}}^{u}$ are then 1-dimensional foliations of the plane.

We now describe four objects which are the main tools in this article.

We say that leaves $F, L \in \widetilde{\mathcal{F}}^{s}$ and $G, H \in \widetilde{\mathcal{F}}^{u}$ form a rectangle if $F$ intersects both $G$ and $H$ and so does $L$, see fig. 2 a. We also say that $E$ intersects $G$ between $F$ and $L$ if $E \cap G$ is contained in the flow band in $G$ defined by $G \cap F$ and $G \cap L$. It is easy to prove that if $E \in \widetilde{\mathcal{F}}^{s}$ intersects $G$ between $F$ and $L$ then $E$ also intersects $H$ between $F$ and $L$. To see this just project to the plane $\mathcal{O}$ : then one sees a true rectangle $[0,1] \times[0,1]$ foliated by horizontal (stable) and vertical (unstable) segments - this last fact follows from index computations of foliations in the plane. Hence there is a product structure of $\widetilde{\mathcal{F}}^{s}$ and $\widetilde{\mathcal{F}}^{u}$ in the region bounded by $F, L, G$ and $H$.

Definition 3.1. Given $p \in \widetilde{M}$ (or $p \in \mathcal{O})$, let

$$
\mathcal{J}_{+}^{u}(p)=\left\{F \in \widetilde{\mathcal{F}}^{s} \mid F \cap \widetilde{W}_{+}^{u}(p) \neq \emptyset\right\},
$$

an open subset of $\mathcal{H}^{s}$. Notice that the leaf $\widetilde{W}^{s}(p) \notin \mathcal{J}_{+}^{u}(p)$. Similarly define $\mathcal{J}_{-}^{u}(p)$, $\mathcal{J}_{+}^{s}(p)$ and $\mathcal{J}_{-}^{s}(p)$. Let also $\mathcal{L}_{+}^{u}(p)=\cup\left\{p \in \widetilde{M} \mid p \in F \in \mathcal{J}_{+}^{u}(p)\right\}$. Then $\mathcal{L}_{+}^{u}(p)$ is an open subset of $\widetilde{M}$ and $\widetilde{W^{s}}(p) \subset \partial \mathcal{L}_{+}^{u}(p)$. Similarly define $\mathcal{L}_{-}^{u}(p), \mathcal{L}_{+}^{s}(p)$ and $\mathcal{L}_{-}^{s}(p)$.

Definition 3.2. Two leaves $F \in \widetilde{\mathcal{F}}^{s}$ and $G \in \widetilde{\mathcal{F}}^{u}$, form a perfect fit $(F, G)$ if $F \cap G=\emptyset$ and there are half leaves $F_{1}$ of $F$ and $G_{1}$ of $G$ and also flow bands $L_{1} \subset L \in \widetilde{\mathcal{F}}^{s}$ and $H_{1} \subset H \in \widetilde{\mathcal{F}}^{u}$, so that:

$$
\bar{L}_{1} \cap \bar{G}_{1}=\partial L_{1} \cap \partial G_{1}, \quad \bar{L}_{1} \cap \bar{H}_{1}=\partial L_{1} \cap \partial H_{1}, \quad \bar{H}_{1} \cap \bar{F}_{1}=\partial H_{1} \cap \partial F_{1},
$$




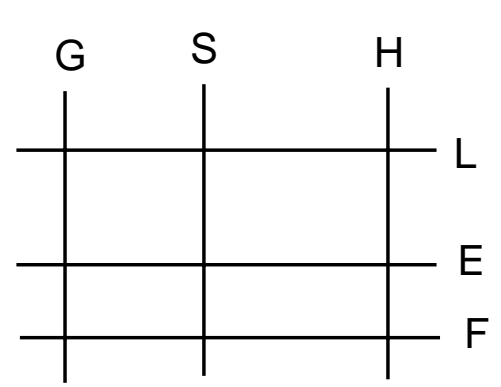

(a)

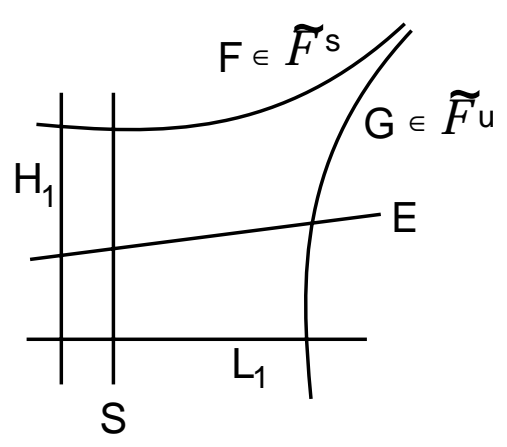

(b)

Figure 2. a. Rectangles, b. Perfect fits in the universal cover.

$$
\begin{gathered}
\forall S \in \widetilde{\mathcal{F}}^{u}, \quad S \cap L_{1} \neq \emptyset \Rightarrow S \cap F_{1} \neq \emptyset \quad \text { (1) and } \\
\forall E \in \widetilde{\mathcal{F}}^{s}, \quad E \cap H_{1} \neq \emptyset \Rightarrow E \cap G_{1} \neq \emptyset \quad \text { (2). }
\end{gathered}
$$

See figure $2 b$. The flow bands $L_{1}, H_{1}$ (or the leaves $L, H$ ) are not uniquely determined by the perfect fit $(F, G)$.

We claim that the implications (1), (2) in fact imply equivalences (that is $S \cap$ $L_{1} \neq \emptyset \Leftrightarrow S \cap F_{1} \neq \emptyset$ and the same for (2)). To see this let $S \in \widetilde{\mathcal{F}}^{u}$ with $S \cap F_{1} \neq \emptyset$. Choose $R \in \widetilde{\mathcal{F}}^{s}$ near enough $F$, so that $R \cap H_{1} \neq \emptyset$ and $R \cap S \neq \emptyset$. By (2), $R \cap G_{1} \neq \emptyset$. If $S=G$ then $G \cap F \neq \emptyset$, contradiction. If $G$ separates $H$ from $S$, then $G$ separates $F$ from $S$, contradiction to $F_{1} \cap S \neq \emptyset$. Hence $S$ intersects $R$ between $G$ and $H$. Since $R, L, G, H$ form a rectangle, this implies that $S$ intersects $L$ between $G$ and $H$, that is, $S$ intersects $L_{1}$. This proves the stronger equivalence in (1) and similarly for (2).

Perfect fits produce "ideal" rectangles, in the sense that even though $F$ and $G$ do not intersect, there is a product structure (of $\widetilde{\mathcal{F}}^{s}$ and $\widetilde{\mathcal{F}}^{u}$ ) in the interior of the region bounded by $F, L, G$ and $H$.

There is at most one leaf $G \in \widetilde{\mathcal{F}}^{u}$ making a perfect fit with a given half leaf of $F \in \widetilde{\mathcal{F}}^{s}$ and in a given side of $F$ [Fe5]. Therefore if $(L, G)$ forms a perfect fit and $g$ is an orientation preserving covering translation with $g(L)=L$, then $g(G)=G$. This follows from uniqueness of perfect fits and the fact that $g$ takes perfect fits to perfect fits, because it acts by homeomorphisms in the leaf spaces.

Definition 3.3. Lozenges - - Suppose $p, q \in \widetilde{M}$ satisfy

$$
\mathcal{J}_{+}^{u}(p) \cap \mathcal{J}_{+}^{s}(p)=\mathcal{J}_{-}^{u}(q) \cap \mathcal{J}_{-}^{s}(q)
$$




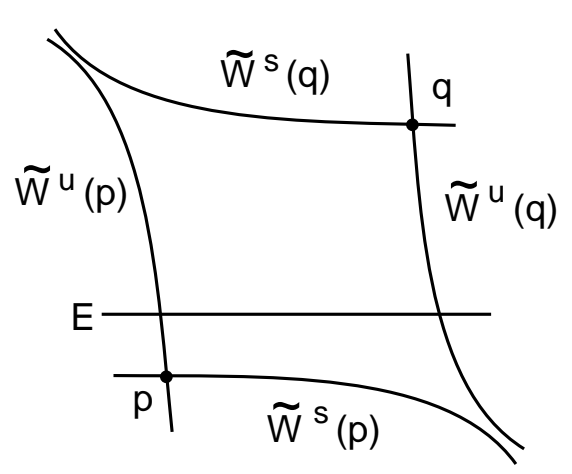

(a)

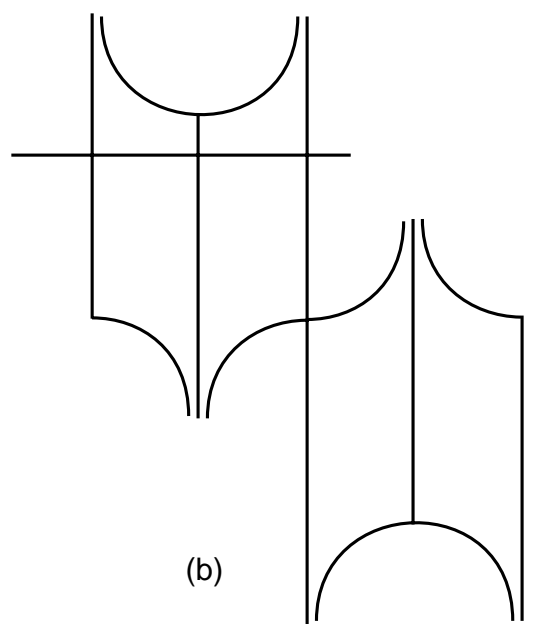

Figure 3. a. A lozenge, b. A chain of lozenges.

Then we say that this intersection is a lozenge $\mathcal{B}$ in $\widetilde{M}$ with $(-,-)$ corner $p$ (or $\left.\widetilde{\Phi}_{\mathbf{R}}(p)\right)$ and $(+,+)$ corner $q$. Notice that equation (i) implies that $\mathcal{J}_{+}^{u}(p)=\mathcal{J}_{-}^{u}(q)$ and $\mathcal{J}_{+}^{s}(p)=\mathcal{J}_{-}^{s}(q)$. If on the other hand $p$ and $q$ satisfy

$$
\mathcal{J}_{+}^{s}(p) \cap \mathcal{J}_{-}^{u}(p)=\mathcal{J}_{-}^{s}(q) \cap \mathcal{J}_{+}^{u}(q) \quad(i i) .
$$

then this intersection is a lozenge $\mathcal{B}$ with $(+,-)$ corner $p$ and $(-,+)$ corner $q$. In any case it follows that $\widetilde{W}^{s}(p), \widetilde{W}^{u}(q)$ form a perfect fit and so do $\widetilde{W}^{s}(q), \widetilde{W}^{u}(p)$ - this is an equivalent way to define a lozenge. The lozenge is an open region in $\widetilde{M}$. The sides of the lozenge in case (i) are $\widetilde{W}_{+}^{s}(p), \widetilde{W}_{+}^{u}(p), \widetilde{W}_{-}^{s}(q)$ and $\widetilde{W}_{-}^{u}(q)$ and are not contained in $\mathcal{B}$, but rather are contained in $\partial \mathcal{B}$. Similarly for case (ii).

Since given any four leaves there is at most one lozenge with sides in them we also sometimes refer to the full leaves as the sides of the lozenge.

Two lozenges are adjacent if they share a corner and there is a stable or unstable leaf intersecting both of them, see fig. 3 b. A chain of lozenges is a collection $\left\{\mathcal{B}_{i}\right\}, i \in I$, where $I$ is an interval (finite or not) in $\mathbf{Z}$; so that if $i, i+1 \in I$, then $\mathcal{B}_{i}$ and $\mathcal{B}_{i+1}$ share a corner see fig. $3 \mathrm{~b}$. Consecutive lozenges may be adjacent or not. The chain is finite if $I$ is finite.

Definition 3.4. Suppose $\eta \subset F \in \widetilde{\mathcal{F}}^{s}$ is a (possibly infinite) strong stable segment so that

$$
\forall p, q \in \eta, \quad \mathcal{J}_{+}^{u}(p)=\mathcal{J}_{+}^{u}(q) . \quad \text { In that case let } \mathcal{P}=\bigcup_{p \in \eta} \widetilde{W}_{+}^{u}(p) .
$$




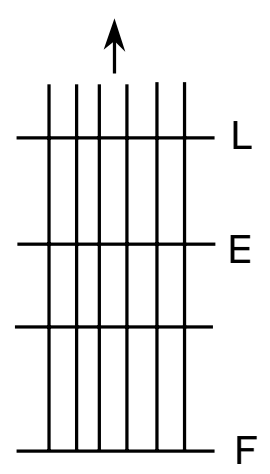

$\eta$

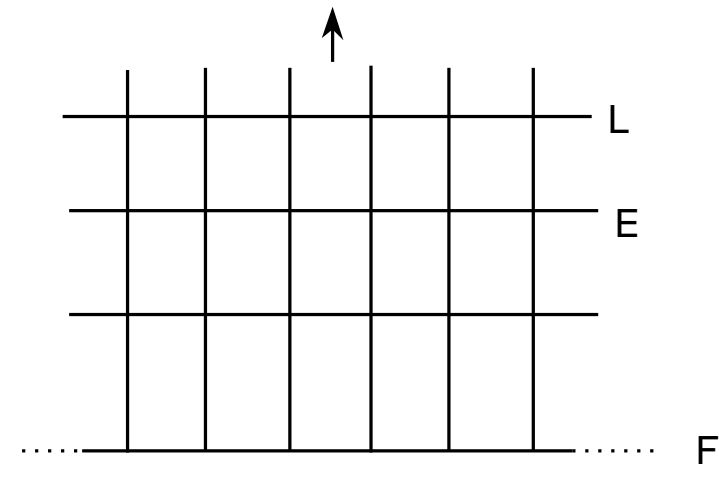

$\eta$

(a)

(b)

Figure 4. a. A product region with bounded base segment, b. A product region with infinite base segment. Horizontals are stable leaves and verticals are unstable leaves. Dots indicate an infinite basis segment. Arrows indicate that unstable leaves extend indefinitely.

Then $\mathcal{P} \subset \widetilde{M}$ is called a positive unstable product region with base segment $\eta$, see fig. 4. The basis segment is not uniquely determined by $\mathcal{P}$. Similarly define negative unstable product regions and stable product regions.

The main property of product regions is the following: for any $F \in \widetilde{\mathcal{F}}^{s}, G \in \widetilde{\mathcal{F}}^{u}$ so that (i) $F \cap \mathcal{P} \neq \emptyset$ and (ii) $G \cap \mathcal{P} \neq \emptyset$, then $F \cap G \neq \emptyset$. To see this, first notice that (ii) implies that $\emptyset \neq G \cap \eta=p$. By (i) let $q \in \eta$ with $F \cap \widetilde{W}_{+}^{u}(q) \neq \emptyset$. Then $F \in \mathcal{J}_{+}^{u}(q)$ hence $F \in \mathcal{J}_{+}^{u}(p)$, that is $F \cap G \neq \emptyset$.

We will also denote by rectangles, perfect fits, lozenges and product regions the projection of these regions to $\mathcal{O}$. One good way to visualize these objects in $\mathcal{O}$ is as follows. Consider proper embeddings $\xi: U \rightarrow \mathcal{O}$ of sets $U \subset \mathbf{R}^{2}$ into $\mathcal{O}$, sending the horizontal and vertical foliations induced in $U$ to the stable and unstable foliations in $\xi(U) \subset \mathcal{O}$. Then a proper embedding is associated to a rectangle $\xi(U)$ if $U=[0,1] \times[0,1]$. A proper embedding is associated to a perfect fit if $U$ is a rectangle without a corner, that is, $U=[0,1] \times[0,1]-\{(1,1)\}$. A lozenge is associated to the image of a rectangle without two opposite corners $U=[0,1] \times[0,1]-\{(1,1),(0,0)\}$ (the lozenge is the interior of $\xi(U)$ ). A stable product region is associated to the image of $U=[a, b] \times[0, \infty)($ or $U=\mathbf{R} \times[0, \infty)$ when the base segment is infinite) and similarly for an unstable product region.

We say that an orbit $\gamma$ of $\widetilde{\Phi}$ is periodic if it is left invariant by a non trivial covering translation. Similarly we define periodic for $F \in \widetilde{\mathcal{F}}^{s}$ or $\widetilde{\mathcal{F}}^{u}$.

If $p, q$ are in the same strong stable leaf let $[p, q]_{s}$ denote the closed segment in that leaf from $p$ to $q$ and let $(p, q)_{s}$ be the corresponding open segment. Similarly define $[p, q]_{u}$ and $(p, q)_{u}$. 


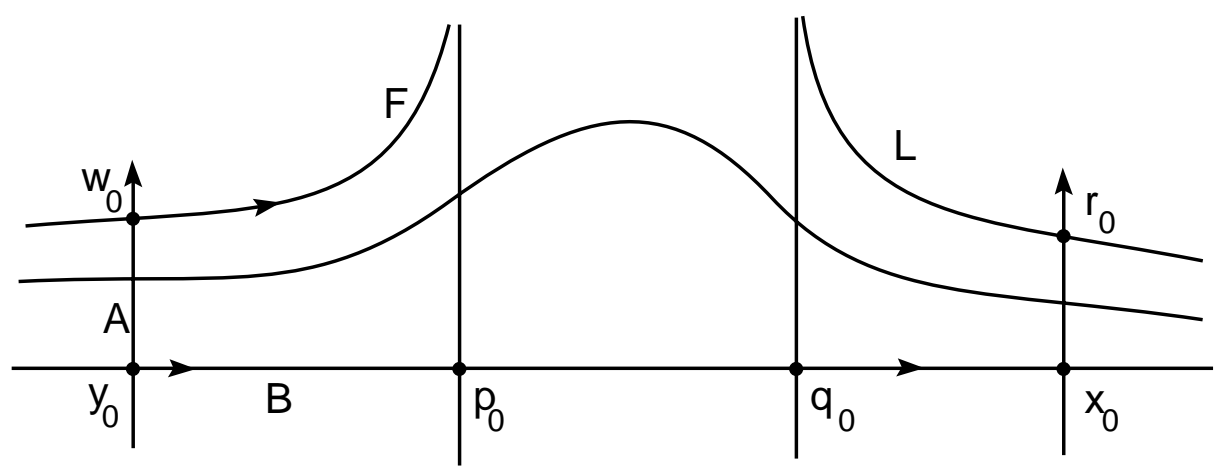

Figure 5. Branching in $\widetilde{\mathcal{F}}^{s}$.

Theorem 3.5. Let $\Phi$ be an Anosov flow in $M^{3}$ and let $F$ be a branching leaf of $\widetilde{\mathcal{F}}^{s}$. Then there is a non trivial covering translation $g$ with $g(F)=F$, that is, $F$ is periodic.

Proof. By taking a finite cover if necessary, we may assume that $\Phi$ is orientable. Let $L \in \widetilde{\mathcal{F}}^{s}, L \neq F$, so that $F, L$ form a branching pair of $\widetilde{\mathcal{F}}^{s}$. Assume without loss of generality that $F$ and $L$ are not separated on their negative sides, that is they are associated to branching of $\widetilde{\mathcal{F}}^{s}$ in the positive direction (positive branching).

Let $w_{0} \in F, w^{\prime} \in L$. Since $F$ and $L$ are not separated in their negative sides there are $y_{0} \in \widetilde{W}_{-}^{u u}\left(w_{0}\right)\left(y_{0}\right.$ sufficiently near $\left.w_{0}\right)$ and $x_{0}=\widetilde{W}^{u}\left(w^{\prime}\right) \cap \widetilde{W}^{s s}\left(y_{0}\right)$ so that if $r_{0}=\widetilde{W}_{+}^{u u}\left(x_{0}\right) \cap L$, then for any $E \in \widetilde{\mathcal{F}}^{s}$,

$$
E \cap\left(y_{0}, w_{0}\right)_{u} \neq \emptyset \Longleftrightarrow E \cap\left(x_{0}, r_{0}\right)_{u} \neq \emptyset .
$$

This fact, which follows from the separation property of leaves of $\widetilde{\mathcal{F}}^{s}$, will often be implicitly used.

Switch $F$ and $L$ if necessary, so that $\widetilde{W}^{u}\left(x_{0}\right)$ is in the front of $\widetilde{W}^{u}\left(y_{0}\right)$. We first find unique leaves associated to the branching, which form perfect fits with $F$ and $L$. As there are $z \in\left[y_{0}, x_{0}\right]_{s}$ with $\widetilde{W}^{u}(z) \cap F=\emptyset$ (e.g. $\left.z=x_{0}\right)$, let $p_{0}$ be the closest point to $y_{0}$ in $\left[y_{0}, x_{0}\right]_{s}$ so that $\widetilde{W}^{u}\left(p_{0}\right) \cap F=\emptyset$.

Claim. The leaves $F$ and $\widetilde{W}^{u}\left(p_{0}\right)$ form a perfect fit.

For flow bands let $A=\widetilde{\Phi}_{\mathbf{R}}\left(\left(y_{0}, w_{0}\right)_{u}\right)$ and $B=\widetilde{\Phi}_{\mathbf{R}}\left(\left(y_{0}, p_{0}\right)_{s}\right)$. Then $\bar{A} \cap \bar{B}=$ $\widetilde{\Phi}_{\mathbf{R}}\left(y_{0}\right), \bar{A} \cap F=\widetilde{\Phi}_{\mathbf{R}}\left(w_{0}\right)$ and $\bar{B} \cap \widetilde{W}^{u}\left(p_{0}\right)=\widetilde{\Phi}_{\mathbf{R}}\left(p_{0}\right)$.

Let $E \in \widetilde{\mathcal{F}}^{s}$ with $E \cap A \neq \emptyset$. Then $E \cap \widetilde{W}^{u}\left(x_{0}\right) \neq \emptyset$. Since $\widetilde{W}^{u}\left(p_{0}\right)$ separates $M$ it follows that $E \cap \widetilde{W}^{u}\left(p_{0}\right) \neq \emptyset$. As $E$ is in front of $\widetilde{W}^{s}\left(y_{0}\right)$ then $E \cap \widetilde{W}_{+}^{u}\left(p_{0}\right) \neq \emptyset$. 
Let $R \in \widetilde{\mathcal{F}}^{u}$ with $R \cap B \neq \emptyset$. If $R \cap F=\emptyset$, then $z=R \cap\left[y_{0}, p_{0}\right]_{s}$ is closer to $y_{0}$ (in $\left.\widetilde{W}^{s s}\left(y_{0}\right)\right)$ than $p_{0}$, contradiction. Hence $R \cap F \neq \emptyset$, in particular $R \cap \widetilde{W}_{+}^{s}\left(w_{0}\right) \neq \emptyset$. By definition it follows that $\widetilde{W}_{+}^{s}\left(w_{0}\right)$ and $\widetilde{W}_{+}^{u}\left(p_{0}\right)$ form a perfect fit. This finishes the proof of the claim.

In the same way there is a unique $q_{0} \in\left[y_{0}, x_{0}\right]_{s}$ with $\widetilde{W}^{u}\left(q_{0}\right)$ and $L$ forming a perfect fit. By uniqueness of perfect fits, the leaves $\widetilde{W}^{u}\left(p_{0}\right), \widetilde{W}^{u}\left(q_{0}\right)$ depend only on $F$ and $L$. If follows from $(*)$ and the claim, that given $E \in \widetilde{\mathcal{F}}^{s}, E \cap \widetilde{W}_{+}^{u}\left(p_{0}\right) \neq$ $\emptyset \Leftrightarrow E \cap \widetilde{W}_{+}^{u}\left(q_{0}\right) \neq \emptyset$. Equivalently $\mathcal{J}_{+}^{u}\left(p_{0}\right)=\mathcal{J}_{+}^{u}\left(q_{0}\right)$.

Case 1. $p_{0}=q_{0}$.

Let $G=\widetilde{W}^{u}\left(p_{0}\right)=\widetilde{W}^{u}\left(q_{0}\right)$. If $G$ is periodic there is $g \neq i d$ with $g(G)=G$. By uniqueness of perfect fits and preservation of transversal orientations $\left(\mathcal{F}^{s}, \mathcal{F}^{u}\right.$ are transversely orientable in the finite cover) it follows that $g(F)=F$ and we are done. Hence assume from now on that $G$ is not periodic.

Let $c_{0}=\pi\left(p_{0}\right)$. Since $G$ is not periodic, $\Phi_{\mathbf{R}}\left(c_{0}\right)$ is not a closed orbit, nor is it backwards asymptotic to a closed orbit. Let $c$ be a negative limit point of $\Phi_{\mathbf{R}}\left(c_{0}\right)$ and let $c_{i}=\Phi_{t_{i}}\left(c_{0}\right), t_{i} \rightarrow-\infty$, with $c_{i} \rightarrow c$. If $c_{i}$ and $c_{j}$ are in the same local unstable leaf near $c$, then there is a closed path in $W^{u}\left(c_{i}\right)$ consisting of the flow segment from $c_{i}$ to $c_{j}$ and a small arc from $c_{j}$ to $c_{i}$ in the local unstable leaf through $c_{j}$. This path is not null homotopic in $W^{u}\left(c_{i}\right)$, hence $W^{u}\left(c_{i}\right)$ contains a closed orbit, contradiction to our assumption. This is the key fact used in the proof of the theorem and it will imply that non periodic leaves in the universal cover are not rigid.

Lift $c_{i}$ to $p_{i} \in \widetilde{M}$ with $p_{i} \rightarrow p$ and $\pi(p)=c$. Then $p_{i}=g_{i}\left(\widetilde{\Phi}_{t_{i}}\left(p_{0}\right)\right)$, where $g_{i}$ are covering translations. By the above argument $\widetilde{W}^{u}\left(p_{i}\right) \neq \widetilde{W}^{u}\left(p_{k}\right)$ for any $i \neq k$. This is the non rigid behavior we are looking for.

Let $F_{i}=g_{i}(F), L_{i}=g_{i}(L), A_{i}=g_{i}(A), B_{i}=g^{i}(B)$ and $G_{i}=g_{i}(G)$. Let $y_{i}=g_{i}\left(\widetilde{\Phi}_{t_{i}}\left(y_{0}\right)\right)$ and let $x_{i}=g_{i}\left(\widetilde{\Phi}_{t_{i}}\left(x_{0}\right)\right)$. Up to subsequence assume that all $p_{i}$ and $p$ are near enough, in a product neighborhood of $\widetilde{\mathcal{F}}^{u}$ of diameter $<<1$. Assume also that for all $i$,

$$
l\left(\widetilde{\Phi}_{t_{i}}\left(\left[y_{0}, p_{0}\right]_{s}\right)\right)>1 \text { and } l\left(\widetilde{\Phi}_{t_{i}}\left(\left[p_{0}, x_{0}\right]_{s}\right)\right)>1 .
$$

Choose indices $i, k$ so that $p_{i}$ is in the back of $\widetilde{W}^{u}\left(p_{k}\right)$, see fig. 6 . Since $d\left(p_{i}, p_{k}\right)<<1$ it follows that $\widetilde{W}_{-}^{s}\left(p_{k}\right) \cap \widetilde{W}^{u}\left(p_{i}\right) \neq \emptyset$ and $\widetilde{W}_{+}^{s}\left(p_{i}\right) \cap \widetilde{W}^{u}\left(p_{k}\right) \neq \emptyset$. By $(* *)$, this implies that $y_{k}$ is in the back of $\widetilde{W}^{u}\left(p_{i}\right)$ and $x_{i}$ is in the front of $\widetilde{W}^{u}\left(p_{k}\right)$, see fig. 6 . Hence $\widetilde{W}^{u}\left(y_{k}\right)$ is in the back of $\widetilde{W}^{u}\left(p_{i}\right)$. Then $\widetilde{W}^{u}\left(p_{i}\right) \cap B_{k} \neq \emptyset$ and by the defining property of perfect fits $\widetilde{W}^{u}\left(p_{i}\right) \cap F_{k} \neq \emptyset$. As $L_{i}$ makes a perfect fit with $\widetilde{W}^{u}\left(p_{i}\right)$, then $L_{i}$ is in front of $F_{k}$, hence $L_{i}$ is in the back of $\widetilde{W}^{u}\left(p_{k}\right)$.

On the other hand, $L_{i} \cap \widetilde{W}^{u}\left(x_{i}\right) \neq \emptyset$. Since $\widetilde{W}^{u}\left(x_{i}\right)$ is in front of $\widetilde{W^{u}}\left(p_{k}\right)$ then $\widetilde{W}^{u}\left(p_{k}\right) \cap \widetilde{\Phi}_{\mathbf{R}}\left(\left[p_{i}, x_{i}\right]_{s}\right) \neq \emptyset$. As $L_{i}$ and $\widetilde{W}^{u}\left(p_{i}\right)$ form a perfect fit, this implies that $\widetilde{W}^{u}\left(p_{k}\right) \cap L_{i} \neq \emptyset$. This contradicts the conclusion of the previous paragraph. 


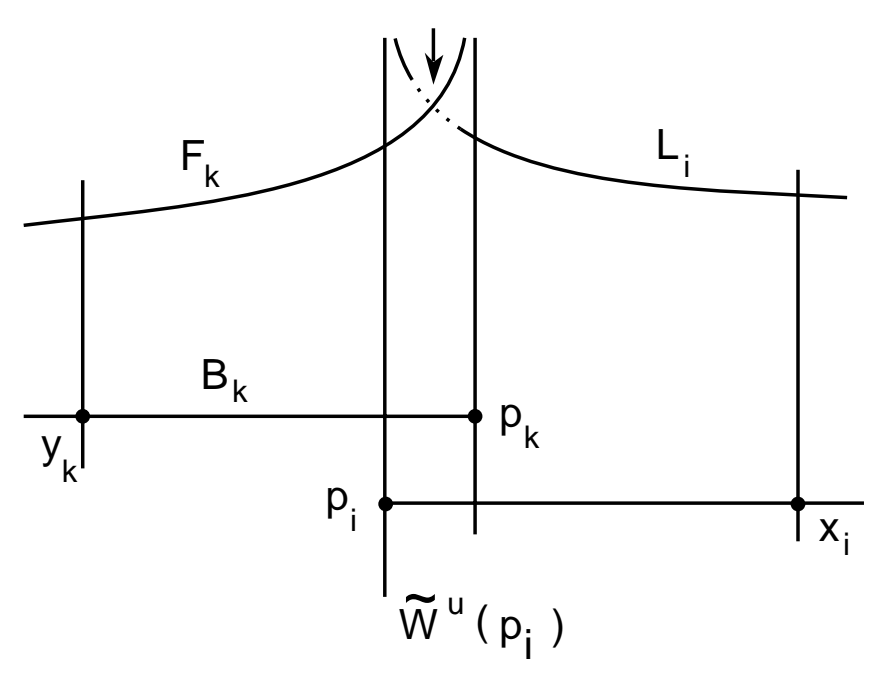

Figure 6. Rigidity of branching leaves: the adjacent case

This shows that if $p_{0}=q_{0}$, then $G$ is periodic, left invariant by $g$, hence $F$ and $L$ are periodic and both left invariant under $g$. This finishws the proof in case 1 .

Remarks. (1) Applying this argument when $G$ is periodic, we get $\widetilde{W}^{s}\left(p_{i}\right)=$ $\widetilde{W}^{s}\left(p_{k}\right) \forall i, k$. There is no small perturbation of the local picture which is then rigid.

(2) It is tempting to try the following "intuitive" approach to the above proof: as $\pi\left(\widetilde{W}_{+}^{u}\left(p_{0}\right)\right)$ is not compact, there are translates $S_{1}$ and $S_{2}$ of $\widetilde{W}^{u}\left(p_{0}\right)$ and points $u_{i} \in S_{i}$ arbitrarily near each other. The problem with this approach is that there is no control of the rest of the picture. For instance we do not know a priori what happens to the respective stable lengths. This is the reason why we fixed an orbit $\Phi_{\mathbf{R}}\left(\pi\left(p_{0}\right)\right)$ and flowed backwards in order to insure that stable lengths are as big as we want. This is also why the proof is done in $\widetilde{M}$ and not in $\mathcal{O}$.

\section{Case 2. $p_{0} \neq q_{0}$.}

We use the same notation as in case 1 . As $q_{0} \neq p_{0}$, let $q_{i}=g_{i}\left(\widetilde{\Phi}_{t_{i}}\left(q_{0}\right)\right)$. Choose $i, k$ with $p_{i}$ in the back of $\widetilde{W}^{u}\left(p_{k}\right)$. As in case $1, \widetilde{W}_{+}^{u}\left(p_{i}\right) \cap F_{k} \neq \emptyset$. There is no a priori contradiction because now $L_{i}$ does not form a perfect fit with $\widetilde{W}^{u}\left(p_{i}\right)$, and in fact $L_{i}$ is probably in the front of $\widetilde{W}^{u}\left(p_{k}\right)$, see fig. 7. Let then

$$
e_{1}=\widetilde{W}^{u}\left(p_{k}\right) \cap \widetilde{W}_{+}^{s s}\left(p_{i}\right), \quad e_{2}=\widetilde{W}^{u}\left(p_{i}\right) \cap \widetilde{W}_{-}^{s s}\left(p_{k}\right) .
$$

Notice that $\widetilde{W}_{+}^{u}\left(p_{k}\right)$ and $F_{k}$ form a perfect fit and $\widetilde{W}_{+}^{u}\left(e_{2}\right) \cap F_{k} \neq \emptyset$. These two facts imply that $\mathcal{J}_{+}^{u}\left(p_{k}\right)<\mathcal{J}_{+}^{u}\left(e_{2}\right)$. In addition the local product structure of $\widetilde{\mathcal{F}}^{s}, \widetilde{\mathcal{F}}^{u}$ 


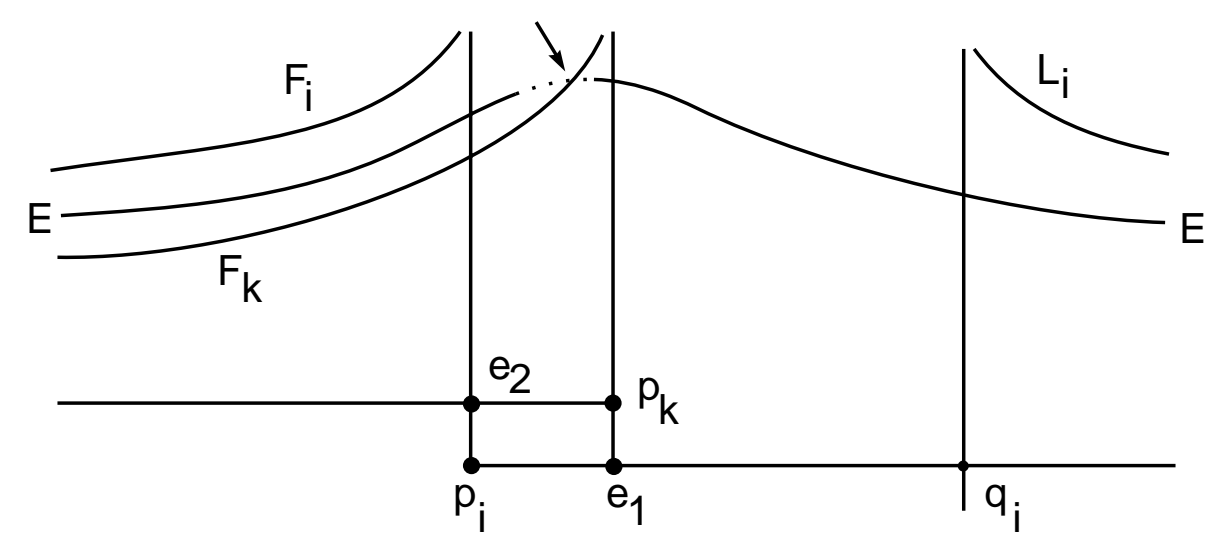

Figure 7. Rigidity of branching: the separated case.

near $p$ implies that $\mathcal{J}_{+}^{u}\left(p_{i}\right)>\mathcal{J}_{+}^{u}\left(e_{1}\right)$, see fig. 7. Choose $E \in \mathcal{J}_{+}^{u}\left(p_{i}\right)-\mathcal{J}_{+}^{u}\left(e_{1}\right)$. By the above considerations it is clear that $E \cap \widetilde{W}^{u}\left(p_{k}\right)=\emptyset$. But

$$
\mathcal{J}_{+}^{u}\left(q_{i}\right)=\mathcal{J}_{+}^{u}\left(g_{i}\left(\widetilde{\Phi}_{t_{i}}\left(q_{0}\right)\right)\right)=g_{i}\left(\mathcal{J}_{+}^{u}\left(\widetilde{\Phi}_{t_{i}}\left(q_{0}\right)\right)\right)=g_{i}\left(\mathcal{J}_{+}^{u}\left(\widetilde{\Phi}_{t_{i}}\left(p_{0}\right)\right)\right)=\mathcal{J}_{+}^{u}\left(p_{i}\right),
$$

hence $E \in \mathcal{J}_{+}^{u}\left(q_{i}\right)$. Then $E \cap \widetilde{W}_{+}^{u}\left(q_{i}\right) \neq \emptyset$. But $\widetilde{W}^{u}\left(q_{i}\right)$ is in the front of $\widetilde{W}^{u}\left(p_{k}\right)$ and as $\widetilde{W}^{u}\left(p_{k}\right)$ separates $\widetilde{M}$, then $E \cap \widetilde{W}^{u}\left(p_{k}\right) \neq \emptyset$. This contradicts the conclusion of the previous paragraph. As before we conclude that $G$ is periodic, left invariant by $g \neq i d$, so $F$ is also left invariant by $g$.

Caution. The same arguments show that $L$ and $\widetilde{W^{u}}\left(q_{0}\right)$ are also periodic. We do not know at this point that the same covering translation leaves invariant both $F$ and $L$. This is a much stronger fact which will be proved in the next section.

\section{Branching structure}

In this section we show that if $F$ and $L$ are not separated, then not only they are periodic, but there is a non trivial covering translation leaving both of them invariant. As an immediate consequence, branching produces a non trivial free homotopy between closed orbits of $\Phi$ in $M$ and this leads to a homotopic characterization of suspensions. We also show that the periodic orbits in $F$ and $L$ are connected by a finite sequence of lozenges. This completely determines the structure of the set of non separated leaves from $F$ and implies that there are only finitely many branching leaves up to covering translations. We then show that if 
there is infinite branching then there is an incompressible torus in $M$ transverse to the flow.

We say that two orbits $\gamma, \alpha$ of $\widetilde{\Phi}$ (or the leaves $\widetilde{W}^{s}(\gamma), \widetilde{W}^{s}(\alpha)$ and similarly for unstable leaves) are connected by a finite chain of lozenges $\left\{\mathcal{B}_{i}\right\}, 1 \leq i \leq n$, if $\gamma$ is a corner of $\mathcal{B}_{1}$ and $\alpha$ is a corner of $\mathcal{B}_{n}$. The following theorem will be essential for the results in this section:

Theorem 4.1. [Fe4] Let $\Phi$ be an Anosov flow in $M^{3}$ and let $F_{0} \neq F_{1} \in \widetilde{\mathcal{F}}^{s}$. Suppose that there is a non trivial covering translation $g$ with $g\left(F_{i}\right)=F_{i}, i=0,1$. Let $\alpha_{i}, i=0,1$ be the periodic orbits of $\widetilde{\Phi}$ in $F_{i}$ so that $g\left(\alpha_{i}\right)=\alpha_{i}$. Then $\alpha_{0}$ and $\alpha_{1}$ are connected by a finite chain of lozenges $\left\{\mathcal{B}_{i}\right\}, 1 \leq i \leq n$ and $g$ leaves invariant each lozenge $\mathcal{B}_{i}$ as well as their corners.

Furthermore there is a unique chain that is minimal, in the sense that any other chain from $\alpha_{0}$ to $\alpha_{1}$ contains this chain [Fe7]. For any chain $\left\{\mathcal{B}_{i}\right\}, 1 \leq i \leq n$ from $\alpha_{0}$ to $\alpha_{1}$, let $\gamma_{0}=\alpha_{0}$ and inductively define $\gamma_{i}, i>0$ to be the remaining corner of $\mathcal{B}_{i}$. The minimal chain from $\alpha_{0}$ to $\alpha_{1}$ is characterized by: $\mathcal{B}_{i+1}$ is on the same side of $\widetilde{W}^{s}\left(\gamma_{i}\right)$ and $\widetilde{W}^{u}\left(\gamma_{i}\right)$ that $\alpha_{1}$ is [Fe7]. Equivalently the lozenges $\left\{\mathcal{B}_{i}\right\}, 1 \leq i \leq n$ are all disjoint $[\mathrm{Fe} 7]$.

A closed orbit of $\Phi$ traversed once is called an indivisible closed orbit.

The following result will be often used in this article:

Theorem 4.2. (Fe7) Let $\Phi$ be an orientable Anosov flow in $M^{3}$. If $\gamma$ is an indivisible closed orbit of $\Phi$, then $\gamma$ represents an indivisible element in $\pi_{1}(M)$. Equivalently if $g$ is a covering translation and $g^{n}(F)=F$, where $F \in \widetilde{\mathcal{F}}^{s}$ or $\widetilde{\mathcal{F}}^{u}$ with $n \neq 0$, then $g(F)=F$.

There is a related result if $\Phi$ is not assumed to be orientable.

The stabilizer $\mathcal{T}(F)$ of a leaf $F$ of $\widetilde{\mathcal{F}}^{s}$ (or $\widetilde{\mathcal{F}}^{u}$ ) is the subgroup of $\pi_{1}(M)$ of those $g$ with $g(F)=F$. If $\pi(F)$ does not contain a periodic orbit, then $\mathcal{T}(F)$ is trivial. Otherwise let $\gamma$ be the indivisible closed orbit in $\pi(F)$. Then $\mathcal{T}(F)$ is infinite cyclic and it has a generator conjugate to $[\gamma]$ in $\pi_{1}(M)$.

The main technical result in this section is theorem $D$ of the introduction:

Theorem 4.3. Let $\Phi$ be an Anosov flow in $M^{3}$. Suppose that $F, L$ form a branching pair of $\tilde{\mathcal{F}}^{s}$. Let $g$ be a non trivial covering translation with $g(F)=F$, so that $g$ preserves transversal orientations to $\widetilde{\mathcal{F}}^{s}, \widetilde{\mathcal{F}}^{u}$. Then $g(L)=L$. Similarly for $\widetilde{\mathcal{F}}^{u}$.

Proof. Up to a cover of order $\leq 4$, assume that $\Phi$ is orientable. As $g$ preserves transversal orientations, then $g$ is still a covering translation of the the universal cover of the finite cover. Assume that $F$ and $L$ are not separated on their negatives sides and also assume that $g$ generates $\mathcal{T}(F)$.

As in theorem 3.5 there are unique leaves $G, H \in \widetilde{\mathcal{F}}^{u}$ making perfect fits with 


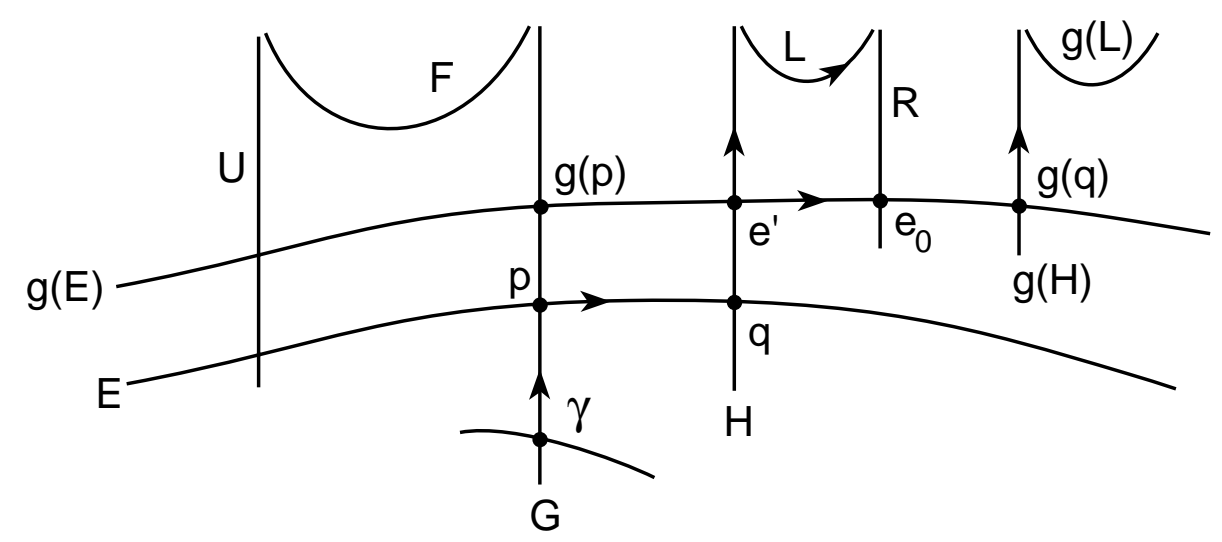

Figure 8. Iterating non invariant leaves.

$F$ and $L$ respectively and so that: $G$ separates $F$ from $L$ and so does $H$. Let $p \in G$ so that $\widetilde{W}^{s s}(p)$ intersects $H$ and let $q=\widetilde{W}^{s s}(p) \cap H$. Recall from the proof of theorem 3.5, that $\mathcal{J}_{+}^{u}(p)=\mathcal{J}_{+}^{u}(q)$. Assume that $H$ is in the front of $G$.

Since $g$ preserves transversal orientations then $g(G)=G$. Our goal is to show that $g(L)=L$. If $G=H$ then this is clear. Suppose then that $G \neq H$ and that $g(L) \neq L$, therefore $g(H) \neq H$. Let $\gamma \subset G$ be the periodic orbit of $\widetilde{\Phi}$ in $G$, so $g(\gamma)=\gamma$.

Claim 1. There is $R \in \widetilde{\mathcal{F}}^{u}$ in the back of $L$ making a perfect fit with a positive half leaf of $L$, hence $R$ is in the front of $H$.

We may assume that $p \in \widetilde{W}_{+}^{u}(\gamma)$. Let $E=\widetilde{W}^{s}(p)$. By taking $g^{-1}$ if necessary assume that $g(E)$ is in front of $E$. Hence $g(E) \in \mathcal{J}_{+}^{u}(p)$, therefore $g(E) \in \mathcal{J}_{+}^{u}(q)$. Then $H \cap g(E) \neq \emptyset$. There are 2 cases:

(1) $g(H)$ is in front of $H$, see fig. 8.

Let Let $e^{\prime}=\widetilde{W}^{s s}(g(p)) \cap H$. Since $g(p) \in \widetilde{W}_{+}^{u}(p)$ then $\mathcal{J}_{+}^{u}(g(p))=\mathcal{J}_{+}^{u}\left(e^{\prime}\right)$. But also $\mathcal{J}_{+}^{u}(g(p))=\mathcal{J}_{+}^{u}(g(q))$, so $\mathcal{J}_{+}^{u}(g(q))=\mathcal{J}_{+}^{u}\left(e^{\prime}\right)$, where $g(q) \in g(H)$ and $e^{\prime} \in H$. Since $L$ makes a perfect fit with $H$ and $g(L)$ makes a perfect fit with $g(H)$ this shows that $g(L)$ is not separated from $L$. As in the proof of theorem 3.5, there is a unique $e_{0} \in\left(e^{\prime}, g(q)\right)_{s}$ with $\widetilde{W}^{u}\left(e_{0}\right)$ making a perfect fit with $L$. In this case let $R=\widetilde{W}^{u}\left(e_{0}\right)$.

(2) Suppose now that $g(H)$ is in the back of $H$.

Notice that $E, g(E), H$ and $G$ form a rectangle. Since $g(H) \cap g(E) \neq \emptyset$ and $g(H)$ is between $G$ and $H$ it follows that $g(H) \cap E \neq \emptyset$ and $g(H) \cap E$ is an orbit in $E$ between $E \cap G$ and $E \cap H$.

In this case let $c=g(H) \cap \widetilde{W}^{s s}(p)$. Then $c \in(p, q)_{s}$. Since $\mathcal{J}_{+}^{u}(p)=\mathcal{J}_{+}^{u}(q)$ and $L \notin \mathcal{J}_{+}^{u}(q)$, then $g(L) \notin \mathcal{J}_{+}^{u}(q)$, so $g(L) \cap H=\emptyset$. Hence $g(L)$ is in the back of $H$. 
As in case (1), it follows that $L$ and $g(L)$ form a branching pair. Let $c_{2} \in(c, q)_{s}$ with $\widetilde{W}^{u}\left(c_{2}\right)$ making a perfect fit with $g(L)$ and with $g(L)$ in the back of $\widetilde{W}^{u}\left(c_{2}\right)$. Then $R=g\left(\widetilde{W}^{u}\left(c_{2}\right)\right)$ makes a perfect fit with $L$ and $L$ is in the back of $\widetilde{W}^{u}\left(c_{2}\right)$. This finishes the proof of claim 1 .

By theorem 3.5, $H, L$ and $R$ are periodic, so let $\alpha, \alpha^{*}$ and $\tau$ be the periodic orbits in $H, L$ and $R$ respectively. Let $h$ a generator of $\mathcal{T}(H)$. Since $\Phi$ is orientable, $h(H)=H, h(R)=R$ and $h(L)=L$.

Claim 2. $\widetilde{W}_{-}^{s}\left(\alpha^{*}\right) \subset L$ and $\widetilde{W}_{+}^{u}(\alpha) \subset H$ are two sides of a lozenge $\mathcal{N}_{1}$ with other sides in $\widetilde{W}_{+}^{s}(\alpha)$ and $\widetilde{W}_{-}^{u}\left(\alpha^{*}\right)$, see fig. 9 .

Let $a \in \alpha, b \in \alpha^{*}$ and $Z \in \widetilde{\mathcal{F}}^{s}$ with $Z \cap \widetilde{W}_{-}^{u}\left(\alpha^{*}\right) \neq \emptyset$. Assume that $h$ is a contraction in the set of orbits of $\widetilde{W}^{u}\left(\alpha^{*}\right)$. Then for $n>0$ big enough $h^{n}(Z) \cap \widetilde{W}_{+}^{u}(\alpha) \neq \emptyset$, because $\widetilde{W}^{u}(\alpha)$ and $\widetilde{W}^{s}\left(\alpha^{*}\right)$ form a perfect fit. Since $\widetilde{W}_{+}^{u}(\alpha)$ is $h$ invariant then $Z \cap \widetilde{W}_{+}^{u}(\alpha) \neq \emptyset$. Using similar arguments one concludes that $\mathcal{J}_{+}^{u}(a)=\mathcal{J}_{-}^{u}(b)$ and $\mathcal{J}_{+}^{s}(a)=\mathcal{J}_{-}^{s}(b)$. Consequently $a$ and $b$ are the corners of a lozenge $\mathcal{N}_{1}$ as claimed.

Equivalently the claim says that if $A_{1} \in \widetilde{\mathcal{F}}^{s}$ and $A_{2} \in \widetilde{\mathcal{F}}^{u}$ form a perfect fit and there is a non trivial covering translation $g^{\prime}$ with $g^{\prime}\left(A_{1}\right)=A_{1}$, then $A_{1}$ and $A_{2}$ are two sides of a lozenge $\mathcal{B}$. The corners of the lozenge are two periodic orbits which are invariant under $g^{\prime}$.

In the same way as in the claim above $\widetilde{W}_{+}^{s}\left(\alpha^{*}\right)$ and $\widetilde{W}_{+}^{u}(\tau)$ are the 2 sides of a lozenge $\mathcal{N}_{2}$. The lozenges $\mathcal{N}_{1}, \mathcal{N}_{2}$ are adjacent and intersect the stable leaf $E$. Let $\mathcal{N}=\mathcal{N}_{1} \cup \mathcal{N}_{2} \cup \widetilde{W}_{-}^{u}\left(\alpha^{*}\right)$, an open connected set.

We now show that $F$ also makes a perfect fit with $U \in \widetilde{\mathcal{F}}^{u}, U \neq G$ and $F$ in the front of $U$, hence $G$ is in the front of $U$, see fig. 9. If $h(G)=G$ then since $g$ generates $\mathcal{T}(G)$, it follows that $h=g^{n}$ for some $n \in \mathbf{Z}-\{0\}$. Theorem 4.2 then implies that $g(H)=H$ contrary to assumption. Hence $h(G) \neq G$. Using claim 1 with the roles of $F, L$ exchanged, we produce the required $U \in \widetilde{\mathcal{F}}^{u}$. As shown in claim 2, there are two adjacent lozenges $\mathcal{D}_{1}$ and $\mathcal{D}_{2}$ with (some) sides in $U, F, G$. Both lozenges intersect a stable leaf which we may assume is $E$. Let $\sigma$ be the periodic orbit in $F$ and $\mathcal{D}=\mathcal{D}_{1} \cup \mathcal{D}_{2} \cup \widetilde{W}_{-}^{u}(\sigma)$.

From now on the proof roughly goes as follows: We will show that $\widetilde{W}^{s}(\gamma)$ intersects $\widetilde{W}_{+}^{u}(\alpha)$ and similarly that $\widetilde{W}^{s}(\alpha)$ intersects $\widetilde{W}_{+}^{u}(\gamma)$, producing a contradiction.

Taking $g^{-1}$ if necessary, suppose that $g(H)$ is in the back of $H$. Let $H_{i}=g^{i}(H)$. Then as in case (2) of claim $1, H_{i+1}$ is in the back of $H_{i}$, and $H_{i} \cap E \neq \emptyset, \forall i \geq 0$. Since $H_{i}$ is always in front of $G$, then $H_{i} \rightarrow S$ with $S \cap E \neq \emptyset$ (and maybe $H_{i}$ also converges to other leaves of $\widetilde{\mathcal{F}}^{u}$ ).

Let $\mathcal{V}_{i}$ be the front of $H_{i}$ and let $\mathcal{V}=\cup_{i \in \mathbf{N}} \mathcal{V}_{i}$. Then $g\left(\mathcal{V}_{i}\right)=\mathcal{V}_{i+1}$ so $g(\mathcal{V})=\mathcal{V}$ and consequently $g(\partial \mathcal{V})=\partial \mathcal{V}$. Since $S \not \subset \mathcal{V}$ it follows that $\partial \mathcal{V}$ is a non empty 


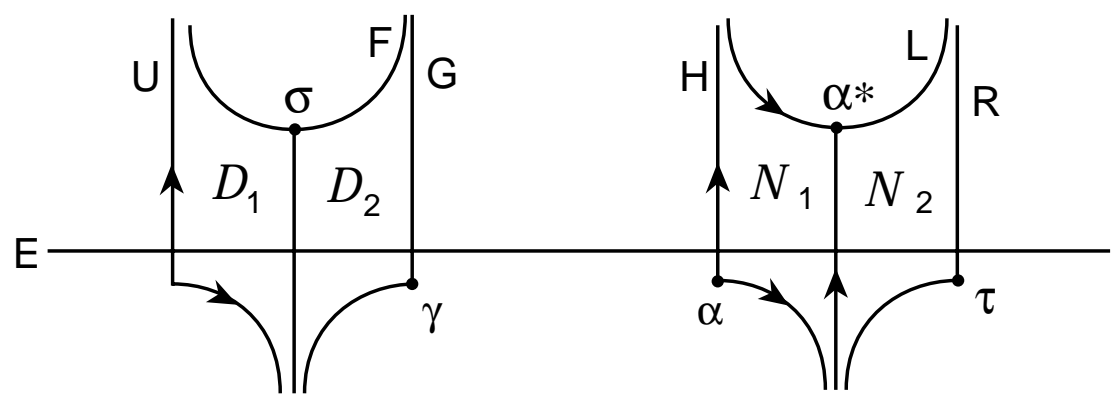

Figure 9. Double lozenges.

union of unstable leaves and furthermore $S \subset \partial \mathcal{V}$. Notice that $S$ is the unique leaf which is either equal to $G$ or separates $G$ from $\mathcal{V}$. In the second case since $g(\mathcal{V})=\mathcal{V}$ and $g(G)=G$ it follows that $g(S)=S$. In either case we have that $g(S)=S$.

Then there is an orbit $\beta$ of $\widetilde{\Phi}$ in $S$ with $g(\beta)=\beta$. By theorem 4.1, $\beta$ and $\gamma$ are connected by a minimal chain of lozenges $\left\{\mathcal{B}_{i}\right\}, 1 \leq i \leq n$.

Furthermore

$$
E \cap S \neq \emptyset, \quad E \cap G \neq \emptyset \Rightarrow E \cap \mathcal{B}_{i} \neq \emptyset, \forall i .
$$

Otherwise choose smallest $i$ so that $E \cap \mathcal{B}_{i} \neq \emptyset$. Then $E \cap \mathcal{B}_{i-1}=\emptyset$ and there is a stable leaf, call it $U$, containing a stable side of $\mathcal{B}_{i}$ and a stable side of $\mathcal{B}_{i-1}$, which separates $E$ from $\mathcal{B}_{i}$. By the characterization of the minimal chain from $\gamma$ to $\beta$, it follows that $U$ separates $E$ from all $\mathcal{B}_{j}, j \geq i$. But $E \cap \mathcal{B}_{n} \neq \emptyset$ because $E \cap S \neq \emptyset$ and $S$ contains a side of $\mathcal{B}_{n}$, contradiction. Therefore consecutive lozenges in the chain $\mathcal{B}_{i}$ are adjacent.

Claim 3. $\gamma$ and all $\mathcal{B}_{i}$ are in the front of $\widetilde{W}^{s}(\beta)$.

Suppose not. Let $r \in \beta$ and $r^{\prime} \in \gamma$. Notice that $p \in \widetilde{W}_{+}^{u}\left(r^{\prime}\right)$. Since $\gamma$ and $\beta$ are connected by a chain of adjacent lozenges all intersecting $E$ and $\gamma$ is in the back of $\widetilde{W}^{s}(\beta)$, it follows that $\mathcal{J}_{-}^{u}(r)=\mathcal{J}_{+}^{u}\left(r^{\prime}\right)$. For all $i$ big enough $\widetilde{W}^{s}(r) \cap H_{i} \neq \emptyset$. Notice that $g^{i}(q) \in H_{i}$. If $g^{i}(q)$ is in front of $\widetilde{W}^{s}(r)$ then $\widetilde{W}^{s}\left(g^{i}(q)\right)$ is in front of $\widetilde{W}^{s}(r)$, contradiction to $\widetilde{W}^{s}\left(g^{i}(q)\right)=\widetilde{W}^{s}\left(g^{i}(p)\right)$ being in the back of $\widetilde{W}^{s}(r)$. Clearly $g_{i}(q) \in \widetilde{W}^{s}(r)$ is also disallowed. The third possible option is that $g_{i}(q)$ is in the back of $\widetilde{W}^{s}(r)$. But then $\widetilde{W}^{s}(r) \in \mathcal{J}_{+}^{u}\left(g^{i}(q)\right)$ and hence $\widetilde{W}^{s}(r) \in \mathcal{J}_{+}^{u}\left(g^{i}(p)\right)$, which is also a contradiction. This shows that $\widetilde{W}^{s}(r) \cap H_{i}=\emptyset$ is impossible. This finishes the proof of claim 3 .

Consequently $\gamma$ is in front of $\widetilde{W}^{s}(\beta)$ and $\gamma, \beta$ are connected by and even number of adjacent lozenges. Therefore $\mathcal{J}_{+}^{u}(r)=\mathcal{J}_{+}^{u}\left(r^{\prime}\right)$.

Since $R_{i}=g^{i}(R)$ separates $H_{i}$ from $H_{i-1}$, then $\widetilde{W}^{s}(\beta) \cap R_{i} \neq \emptyset$ and $\widetilde{W}^{s}(\beta) \cap$ $H_{i} \neq \emptyset$, for $i$ big enough. But $g^{i}\left(\widetilde{W}^{s}(\beta)\right)=\widetilde{W}^{s}(\beta)$, therefore $\widetilde{W}^{s}(\beta) \cap R \neq \emptyset$ and 


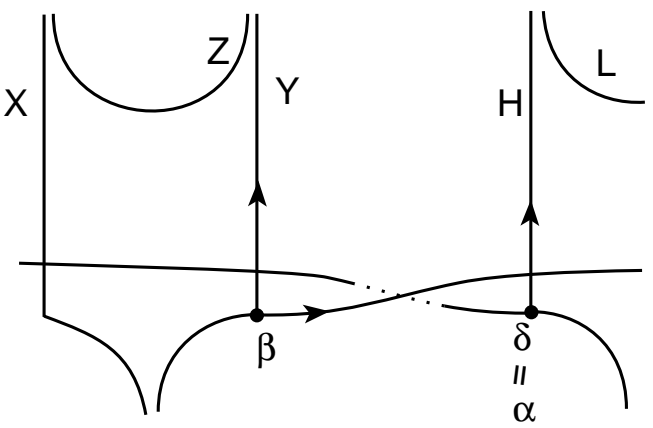

(a) (b)

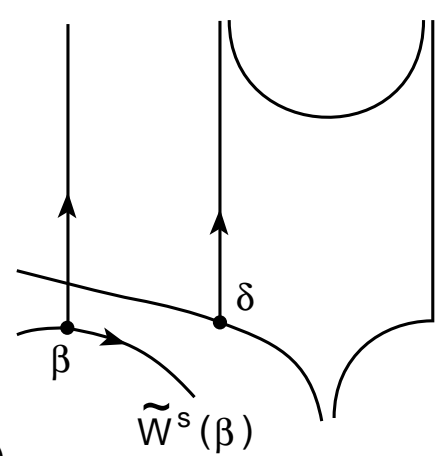

Figure 10. Impossible intersection of leaves: a. Case $\delta=\alpha$, b. Case $\delta \neq \alpha$.

$\widetilde{W}^{s}(\beta) \cap H \neq \emptyset$. Consequently $\left.\widetilde{W}^{s}(\beta)\right) \cap \mathcal{N} \neq \emptyset$ and as a result $\widetilde{W}^{s}(\beta)$ intersects $\widetilde{W}_{+}^{u}(\alpha)$.

Conclusion. There is an orbit $\beta$ of $\widetilde{\Phi}$ with $g(\beta)=\beta, \widetilde{W}^{s}(\beta) \cap \widetilde{W}_{+}^{u}(\alpha) \neq \emptyset$ and $\widetilde{W}^{s}(\beta) \cap R \neq \emptyset$.

Because $\gamma$ and $\beta$ are connected by lozenges $\mathcal{B}_{i}$ all intersecting $E \in \widetilde{\mathcal{F}}^{s}$, then there is $Z \in \widetilde{\mathcal{F}}^{s}$ making a perfect fit with $Y=\widetilde{W}^{u}(\beta)$ so that $Z$ is in the back of $Y$ and $Z$ and $L$ are not separated, see fig. 10 a. Hence $Z, L$ satisfy the hypothesis of this theorem. As in claim 1 there is $X \in \widetilde{\mathcal{F}}^{u}, X \neq Y, X$ making a perfect fit with $Z$ and intersecting $E$, see fig. 10 a. Therefore we could have started the analysis with $Z$ instead of $F$ and $Y$ instead of $G$, considering the orbits $\beta \subset Y$ and $\alpha \subset H$.

Now switch the roles of $Y$ and $H$ and apply the same arguments as above. Then as in the conclusion above we find an orbit $\delta$ of $\widetilde{\Phi}$ with $h(\delta)=\delta$ and $\widetilde{W}^{s}(\delta) \cap \widetilde{W}_{+}^{u}(\beta) \neq \emptyset, \widetilde{W}^{s}(\delta) \cap X \neq \emptyset$. In addition $\delta$ is connected to $\alpha$ by an even chain of lozenges all intersecting a common stable leaf. Hence if $u \in \delta, u^{\prime} \in \alpha$, then $\mathcal{J}_{+}^{u}(u)=\mathcal{J}_{+}^{u}\left(u^{\prime}\right)$.

If $\delta=\alpha$ this produces an immediate contradiction since $\widetilde{W}^{s}(\beta)$ intersects $\widetilde{W}_{+}^{s}(\alpha)$ and $\widetilde{W}^{s}(\alpha)$ intersects $\widetilde{W}_{+}^{s}(\beta)$, see fig. $10 \mathrm{a}$.

Suppose that $\delta \neq \alpha$. As $\widetilde{W}^{s}(\delta) \cap \widetilde{W}_{+}^{u}(\beta) \neq \emptyset$, then $\widetilde{W}^{s}(\beta)$ is in the back of $\widetilde{W}^{s}(\delta)$. In particular $\widetilde{W}^{s}(\beta) \notin \mathcal{J}_{+}^{u}(u)$. Hence $\widetilde{W}^{s}(\beta) \notin \mathcal{J}_{+}^{u}\left(u^{\prime}\right)$, a contradiction to the fact that $\widetilde{W}^{s}(\beta)$ intersects $\widetilde{W}_{+}^{u}(\alpha)$, see fig. $10 \mathrm{~b}$.

This contradiction implies that the assumption $g(H) \neq H$ is impossible to hold. We conclude that $g(H)=H$ and consequently that $g(L)=L$. This finishes the proof of the theorem.

Corollary 4.4. Let $\Phi$ be an Anosov flow in $M^{3}$. Suppose $\Phi$ is not $\mathbf{R}$-covered. Let $F, L \in \widetilde{\mathcal{F}}^{s}$ which are not separated from each other. Then $F$ and $L$ are connected 


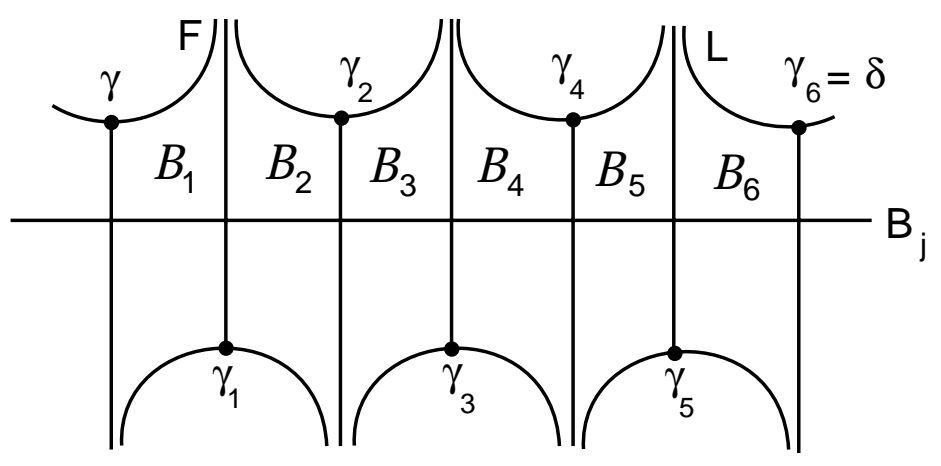

Figure 11. The correct picture of in between branching.

by an even chain of lozenges, all intersected by a common stable leaf. In particular there are only finitely many branching leaves between $F$ and $L$.

Proof. Up to finite covers we may assume that $\Phi$ is orientable. Suppose that $F, L$ are not separated in their negative sides.

Let $g \neq i d$ be a covering translation with $g(F)=F$. By the previous theorem $g(L)=L$. Let $\gamma$ and $\delta$ be the respective periodic orbits in $F$ and $L$. Furthermore suppose $\widetilde{W}^{u}(\gamma)$ is in the back of $\widetilde{W}^{u}(\delta)$.

By theorem 4.1, $\gamma$ and $\delta$ are connected by a chain of lozenges $\left\{\mathcal{B}_{i}\right\}, 1 \leq i \leq n$, assumed to be the minimal chain from $\delta$ to $\gamma$. Let $\gamma_{0}=\gamma$ and inductively define $\gamma_{i}$ to be the other corner of $\mathcal{B}_{i}$. Since $\delta$ is in the back of $\widetilde{W}^{s}(\gamma)$ and in the front of $\widetilde{W}^{u}(\gamma)$ it follows that $\gamma$ is the $(+,-)$ corner of $\mathcal{B}_{1}$. Then $\delta$ is in front of $\widetilde{W}^{s}\left(\gamma_{1}\right)$ and in front of $\widetilde{W}^{u}\left(\gamma_{1}\right)$, hence $\mathcal{B}_{2}$ has $(-,-)$ corner $\gamma_{1}$. Hence $\mathcal{B}_{1}, \mathcal{B}_{2}$ are adjacent, intersecting the same stable leaves and $\widetilde{W}^{s}\left(\gamma_{2}\right)$ is non separated from $\widetilde{W}^{s}\left(\gamma_{0}\right)$ and $\widetilde{W}^{s}(\delta)$. Induction then shows that $\gamma_{i-1}$ is the $(+,-)$ corner of $\mathcal{B}_{i}$ if $i$ is odd, and the $(-,-)$ corner of $\mathcal{B}_{i}$ otherwise. In addition $\widetilde{W}^{s}\left(\gamma_{i}\right)$ is non separated from $\widetilde{W}^{s}\left(\gamma_{0}\right)$ if and only if $i$ is even, hence $n=2 k$.

Suppose now that $E \in \widetilde{\mathcal{F}}^{s}$ is not separated from $F$ and is between $F$ and $L$. Let $\left\{X_{k}\right\}, k \in \mathbf{N} \subset \widetilde{\mathcal{F}}^{s}$, with $X_{k} \rightarrow F$ as $k \rightarrow \infty$. Then $X_{k} \rightarrow E$ in $\mathcal{H}^{s}$ when $k \rightarrow \infty$. In addition for $k$ big $X_{k} \cap \mathcal{B}_{i} \neq \emptyset$ for $1 \leq i \leq n$. Therefore the only possible leaves in the limit of $X_{k}$ which are between $F$ and $L$ are those in the stable boundary of the lozenges $\mathcal{B}_{i}$. This completely characterizes such leaves and hence there are finitely many in between leaves.

An R-covered Anosov flow can only have one of two topological types (up to isotopy in $\widetilde{M}$ ) for the joint structure of $\widetilde{\mathcal{F}}^{s}, \widetilde{\mathcal{F}}^{u}[\mathrm{Fe} 3]$. They are characterized by: 
(1) Any leaf of $\widetilde{\mathcal{F}}^{s}$ intersects every leaf of $\widetilde{\mathcal{F}}^{u}$. This is the product type.

(2) There is a leaf of $\widetilde{\mathcal{F}}^{s}$ which does not intersect every leaf of $\widetilde{\mathcal{F}}^{u}$. This is the skewed type, see detailed characterization in [Fe3].

Suspensions have product type and geodesic flows have skewed type.

Corollary 4.5. Let $\Phi$ be an Anosov flow in $M^{3}$. Then $\Phi$ is topologically conjugate to a suspension of an Anosov diffeomorphism of the torus if and only if there are no free homotopies between closed orbits of $\Phi$ (including non trivial free homotopies from an orbit to itself).

Proof. If $\Phi$ is not R-covered, theorem 4.3 produces $F_{0} \neq F_{1} \in \widetilde{\mathcal{F}}^{s}$ and $g$ a nontrivial covering translation with $g\left(F_{i}\right)=F_{i}$. If $\alpha_{i}$ is the periodic orbit in $F_{i}$, then $g\left(\alpha_{i}\right)=$ $\alpha_{i}$. Therefore $\pi\left(\alpha_{0}\right), \pi\left(\alpha_{1}\right)$ are closed orbits of $\Phi$ (they may be the same orbit) which are non trivially freely homotopic to each other. If $\pi\left(\alpha_{0}\right), \pi\left(\alpha_{1}\right)$ are the same orbit of $\Phi$ in $M$, then the free homotopy is non trivial because $\alpha_{0}, \alpha_{1}$ are distinct orbits of $\widetilde{\Phi}$ in $\widetilde{M}$.

Suppose now that $\Phi$ is $\mathbf{R}$-covered. If $\Phi$ has product type, then by theorem 2.8 of [Ba2] (see announcemment in [So]) $\Phi$ is topologically conjugate to a suspension. Otherwise $\Phi$ has skewed type and theorem 3.4 of [Fe3] produces many non trivial free homotopies between closed orbits of $\Phi$.

Given 2 adjacent lozenges $\mathcal{B}_{1}$ and $\mathcal{B}_{2}$ the pivot of their union is the common corner of $\mathcal{B}_{1}$ and $\mathcal{B}_{2}$.

Corollary 4.6. Let $\Phi$ be an Anosov flow in $M^{3}$. Then up to covering translations there are only finitely many branching leaves.

Proof. Assume that there are infinitely many inequivalent stable branching leaves, where the associated branching is in the positive direction. Given any two non separated leaves $F, L$ let $\gamma, \alpha$ be the respective periodic orbits which are connected by a chain of adjacent lozenges. For any two adjacent lozenges, the pivot is uniquely determined, furthermore the pivots are always periodic orbits.

It follows that there are infinitely many inequivalent periodic pivots $p_{i}, i \in \mathbf{N}$. Since $\pi\left(p_{i}\right)$ accumulates in $M$, assume up to covering translations that all $p_{i}$ are in a very small product neighborhood of $p \in \widetilde{M}$, so let $i \neq k$ with

$$
\widetilde{W}^{u}\left(p_{i}\right) \cap \widetilde{W}^{s}\left(p_{k}\right) \neq \emptyset \text { and } \widetilde{W}^{s}\left(p_{i}\right) \cap \widetilde{W}^{u}\left(p_{k}\right) \neq \emptyset .
$$

An argument exactly as in case 1 of theorem 3.5 shows this is impossible.

We can now completely characterize the structure of the set of non separated leaves: Given $F \in \widetilde{\mathcal{F}}^{s}$, let $\mathcal{E}_{b}(F)$ be the set of leaves of $\widetilde{\mathcal{F}}^{s}$ non separated from $F$ and which are either equal to $F$ or contained in the back of $F$. Similarly define $\mathcal{E}_{f}(F)$. 
Corollary 4.7. Let $\Phi$ be an Anosov flow in $M^{3}$. Let $F$ be a branching leaf of $\widetilde{\mathcal{F}}^{s}$ and $\mathcal{E}_{b}(F)$ be the set of leaves of $\widetilde{\mathcal{F}}^{s}$ which are non separated from $F$ and which are either equal to $F$ or in the back of $F$. If $E \neq L \in \mathcal{E}_{b}(F)$ we say that $E<L$ in $\mathcal{E}_{b}(F)$ if there are $G, H \in \widetilde{\mathcal{F}}^{u}$, with $G \cap E \neq \emptyset, H \cap L \neq \emptyset$ and $G$ in the back of $H$. Then $<$ is a well defined relation in $\mathcal{E}_{b}(F)$ which is a total order and with this order either

(1) $\mathcal{E}_{b}(F)$ is finite, hence order isomorphic to $\{1,2, \ldots, n\}$, or

(2) $\mathcal{E}_{b}(F)$ if infinite and order isomorphic to the set of integers $\mathbf{Z}$. Similarly for $\mathcal{E}_{f}(F)$.

Proof. Up to finite cover if necessary assume that $\Phi$ is orientable. Given $E \neq L \in$ $\mathcal{E}_{b}(F)$, let $G, H \in \widetilde{\mathcal{F}}^{u}$ with $G \cap E \neq \emptyset$ and $H \cap L \neq \emptyset$. Since $E, L$ form a branching pair, there is $A \in \widetilde{\mathcal{F}}^{s}$ intersecting both $G$ and $H$. Therefore either $G$ is in the back of $H$ and $H$ is in the front of $G$ or vice versa (for arbitrary leaves of $\widetilde{\mathcal{F}}^{u}$, it can happen that $G$ is in the front of $H$ and $H$ is in the front of $G$ ). Consequently either $E<L$ in $\mathcal{E}_{b}(F)$ or $L<E$ in $\mathcal{E}_{b}(F)$. It is easy to check that the outcome is independent of the choices of $G, H$ and $A$. Hence $<$ is well defined and all distinct points in $\mathcal{E}_{b}(F)$ are related to each other. Finally it is easy to see that $<$ is an order relation. This shows that $<$ is a total order in $\mathcal{E}_{b}(F)$.

Since $<$ is a total order in $\mathcal{E}_{b}(F)$ then if $\mathcal{E}_{b}(F)$ is finite case (1) holds. Hence assume that $\mathcal{E}_{b}(F)$ is infinite.

Since $\mathcal{E}_{b}(F)$ is infinite corollary 4.6 implies that there are $E^{\prime} \neq E^{*} \in \mathcal{E}_{b}(F)$ and $f$ a covering translation with $f\left(E^{\prime}\right)=E^{*}$. Assume that $E^{\prime}<E^{*}$ in the ordering of $\mathcal{E}_{b}(F)$. Corollary 4.4 implies that $E^{\prime}, E^{*}$ are connected by a finite chain of adjacent lozenges with positive stable boundaries in $E_{0}=E^{\prime}, E_{1}, \ldots, E_{n}=f\left(E_{0}\right)=E^{*} \in$ $\widetilde{\mathcal{F}}^{s}$. Then $E_{i} \in \mathcal{E}_{b}(F), 0 \leq i \leq n$. Clearly $E_{i}<E_{j}$ if $i<j$. Since $E_{0}$ is not separated from $E_{n}$, then $f\left(E_{0}\right)=E_{n}$ is not separated from $f\left(E_{n}\right)$. This produces $E_{n+1}, \ldots, E_{2 n}=f\left(E_{n}\right)$. Using $f^{i}, i \in \mathbf{Z}$, one constructs $\left\{E_{i}\right\}_{i \in \mathbf{Z}} \subset \mathcal{E}_{b}(F)$. If $f^{i}(E)=E$ for some $i \neq 0$, then $f(E)=E$ contradiction. This easily implies that the $\left\{E_{i}\right\}_{i \in \mathbf{Z}}$ are all distinct.

Let now $E \in \mathcal{E}_{b}(F)$. Then $E$ and $E_{0}$ are not separated, hence connected by a minimal finite chain of adjacent lozenges $\mathcal{C}_{i}, 1 \leq i \leq 2 \mathrm{~m}$, all intersecting a common stable leaf and having an even number $2 m$ of lozenges. Suppose that $E_{0}<E$ in $\mathcal{E}_{b}(F)$. Consider now the chain $\mathcal{B}_{i}, 1 \leq i \leq 2 m$ as constructed in corollary 4.4. Notice that the lozenges in a chain are completely determined by a corner plus a direction. Since both chains $\mathcal{C}_{i}$ and $\mathcal{B}_{i}$ consist of consecutively adjacent lozenges, all of which intersect a common stable leaf, it follows that $\mathcal{C}_{i}=\mathcal{B}_{i}$ for all $i$. Consequently $E=E_{m}$. Hence $\mathcal{E}_{b}(F)=\left\{E_{i}\right\}_{i \in \mathbf{Z}}$ and $\mathcal{E}_{b}(F)$ is order isomorphic to $\mathbf{Z}$ as desired.

Notice that any covering translation $f$ conjugates the stabilizers of $F$ and $f(F)$ that is $f \circ(\mathcal{T}(F)) \circ f^{-1}=\mathcal{T}(f(F))$. Therefore conjugation by $f$ takes a generator of $\mathcal{T}(F)$ to a generator of $\mathcal{T}(f(F))$. 
Corollary 4.8. Let $\Phi$ be an Anosov flow in $M^{3}$ orientable. Suppose that $\left\{F_{i}\right\}_{i \in \mathbf{N}} \subset$ $\widetilde{\mathcal{F}}^{s}$ is a collection of distinct leaves, so that $F_{i}$ is not separated from $F_{i+1}$ for all $i$. Then $M$ has an incompressible torus.

Proof. There is a finite cover $M_{2}$ of $M$ where the lifted flow is orientable. By corollary 4.6 there is a covering translation $f$ of $M_{2}$ with $f\left(F_{i}\right)=F_{j}$ and $i<j$.

Let $g$ be a generator of $\mathcal{T}\left(F_{i}\right)$. Then $f g f^{-1}$ generates $\mathcal{T}\left(F_{j}\right)$. Since $F_{i}, F_{i+1}, \ldots$, $F_{j}$ are not separated one from the next, then repeated application of theorem 4.3 shows that $g\left(F_{j}\right)=F_{j}$. By theorem $4.2, g$ is indivisible in $\pi_{1}\left(M_{2}\right)$, so $g$ also generates $\mathcal{T}\left(F_{j}\right)$. Hence either $f g f^{-1}=g$ or $f g f^{-1}=g^{-1}$.

In the first case $f$ and $g$ generate an abelian subgroup of $\pi_{1}\left(M_{2}\right)$. If $f^{n} g^{m}=1$, then $f^{n} g^{m}\left(F_{i}\right)=F_{i}$ hence $f^{n}\left(F_{i}\right)=F_{i}$. If $n \neq 0$ theorem 4.2 implies that $f\left(F_{i}\right)=F_{i}$, contradiction to $F_{i} \neq F_{j}$. Hence $n=0$. Since no multiple of a closed orbit is null homotopic, them $g^{m}=i d$ implies that $m=0$ also. Hence there is a $\mathbf{Z} \oplus \mathbf{Z}$ subgroup of $\pi_{1}\left(M_{2}\right)$. If on the other hand $f g f^{-1}=g^{-1}$, then $f^{2}$ and $g$ generate an abelian subroup of $\pi_{1}\left(M_{2}\right)$ and the same argument produces $\mathbf{Z} \oplus \mathbf{Z}<\pi_{1}\left(M_{2}\right)$.

We conclude that there is a $\mathbf{Z} \oplus \mathbf{Z}$ subgroup of $\pi_{1}(M)$. By the torus theorem [Ga] (which uses $M$ being orientable), either $M$ is a Seifert fibered space or there is an embedded incompressible torus. In the first case, Ghys [Gh] proved that $\Phi$ is up to finite covers, topologically conjugate to a geodesic flow. But then $\Phi$ would be $\mathbf{R}$-covered, contrary to hypothesis. In the second case $M$ is toroidal as desired.

Notice that corollary 4.8 does not assume that all $\left\{F_{i}\right\}$ are non separated from each other, which in fact may not be the case. Consider for instance the first set of examples of intransitive Anosov flows constructed by Franks and Williams in [Fr-Wi]. Then each such flow has a sequence of distinct leaves $F_{i}$ so that $F_{i}$ is not separated from $F_{i+1}$ on their negative sides if $i$ is even, otherwise non separated on their positive sides, see description in the end of section 5 . Therefore $F_{i}$ is separated from $F_{j}$ if $|i-j| \geq 2$.

If on the other hand one assumes that all $\left\{F_{i}\right\}, i \in \mathbf{N}$ are non separated from each other (infinite branching), then we can prove a much stronger result, using only the topological structure of $\widetilde{\mathcal{F}}^{s}, \widetilde{\mathcal{F}}^{u}$ :

Theorem 4.9. Let $\Phi$ be an Anosov flow in $M^{3}$ orientable. If there is infinite branching in (say) $\widetilde{\mathcal{F}}^{s}$ then there is an embedded torus transverse to $\Phi$.

Proof. Assume first that $\Phi$ is orientable. Let $\mathcal{E}=\left\{E_{i}\right\}_{i \in \mathbf{Z}}$ be an infinite, ordered collection of non separated leaves, (say) not separated on their negative sides. Let $\gamma_{i}$ be the periodic orbit in $E_{i}$. By corollary 4.4 , every $E_{i}$ forms part of the boundary of two lozenges: let $\mathcal{B}_{2 i-1}$ be the lozenge with $(+,+)$ corner $\gamma_{i}$ and let $\mathcal{B}_{2 i}$ be the lozenge with $(+,-)$ corner $\gamma_{i}$. Let $F_{i} \in \widetilde{\mathcal{F}}^{s}$ be the other leaf in the 


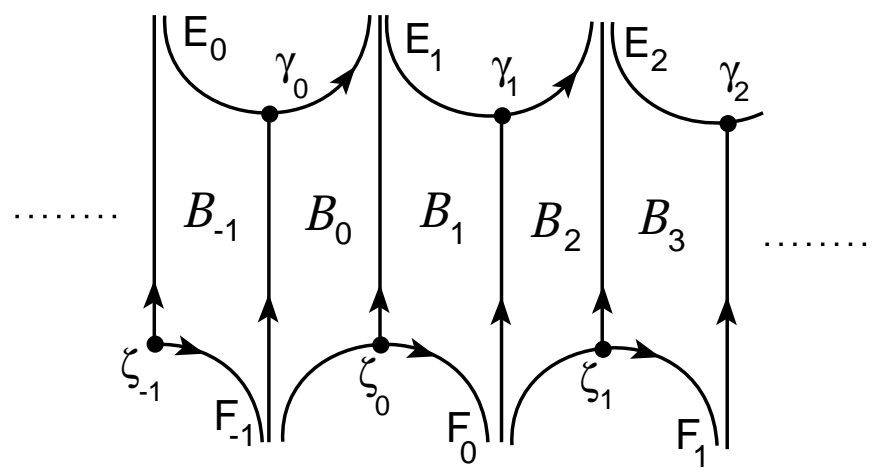

Figure 12. Part of an infinite chain of lozenges.

boundary of $\mathcal{B}_{2 i}$ and $\mathcal{B}_{2 i+1}$, where $\mathcal{B}_{2 i}$ and $\mathcal{B}_{2 i+1}$ are in front of $F_{i}$. Let $\zeta_{i}$ be the periodic orbit in $F_{i}$, see fig. 12. Let

$$
\mathcal{S}=\bigcup_{i \in \mathbf{Z}}\left(\mathcal{B}_{i} \cup \widetilde{W}_{-}^{u}\left(\gamma_{i}\right) \cup \widetilde{W}_{+}^{u}\left(\zeta_{i}\right)\right)
$$

and let $\widetilde{\mathcal{F}}_{\mathcal{S}}^{s}, \widetilde{\mathcal{F}}_{\mathcal{S}}^{u}$ be the restrictions of $\widetilde{\mathcal{F}}^{s}, \widetilde{\mathcal{F}}^{u}$ to $\mathcal{S}$.

Then all of the following sets are equal: $\mathcal{J}_{-}^{u}\left(\gamma_{i}\right), i \in \mathbf{Z}, \quad \mathcal{J}_{+}^{u}\left(\zeta_{j}\right), j \in \mathbf{Z}$. This shows that any leaf of $\widetilde{\mathcal{F}}_{\mathcal{S}}^{s}$ intersects $\widetilde{W}_{-}^{u}\left(\gamma_{0}\right)$, hence the leaf space of $\widetilde{\mathcal{F}}_{\mathcal{S}}^{s}$ is homeomorphic to $\mathbf{R}$. Similarly every leaf of $\widetilde{\mathcal{F}}_{\mathcal{S}}^{u}$ either intersects one of the lozenges $\mathcal{B}_{i}$ or is one of $\widetilde{W}_{-}^{u}\left(\gamma_{i}\right)$ or $\widetilde{W}_{-}^{u}\left(\zeta_{i}\right)$. So the leaf space of $\widetilde{\mathcal{F}}_{\mathcal{S}}^{u}$ is also $\mathbf{R}$. In addition any leaf of $\widetilde{\mathcal{F}}_{\mathcal{S}}^{s}$ intersects every leaf of $\widetilde{\mathcal{F}}_{\mathcal{S}}^{u}$ and vice versa. We conclude that $\Theta(\mathcal{S})$ is homeomorphic to a plane, and up to isotopy $\widetilde{\mathcal{F}}_{\mathcal{S}}^{s}, \widetilde{\mathcal{F}}_{\mathcal{S}}^{u}$ are the foliations by horizontals and verticals in this plane, see fig. 13.

We can now apply corollary 4.8 to produce non trivial commuting $f, g$, so that $g\left(\gamma_{i}\right)=\gamma_{i}, \forall i \in \mathbf{Z}$ and $f\left(\gamma_{0}\right)=\gamma_{k}$ for some $k \neq 0$. Clearly both $f$ and $g$ preserve $\mathcal{S}$. Let $f_{*}, g_{*}$ be the induced actions in $\Theta(\mathcal{S})$.

Let $p \in \Theta\left(\widetilde{W}_{-}^{u}\left(\gamma_{0}\right)\right)$ and $q=f_{*}(p) \in \Theta\left(\widetilde{W}_{-}^{u}\left(\gamma_{k}\right)\right)$. Given the structure of $\Theta\left(\widetilde{\mathcal{F}}_{\mathcal{S}}^{s}\right)$ and $\Theta\left(\widetilde{\mathcal{F}}_{\mathcal{S}}^{u}\right)$ in $\Theta(\mathcal{S})$, it follows that we can connect $p$ and $q$ by a curve $\alpha$ in $\mathcal{O}$, with $\alpha$ always transverse to $\Theta\left(\widetilde{\mathcal{F}}_{\mathcal{S}}^{s}\right)$ and $\Theta\left(\widetilde{\mathcal{F}}_{\mathcal{S}}^{u}\right)$. Let $p_{1} \in \widetilde{M}$ with $\Theta\left(p_{1}\right)=p$ and lift $\alpha$ to $\alpha_{1}$ starting at $p_{1}$ and ending at $q_{1}=f\left(p_{1}\right)$. We may assume that $\alpha_{1}$ is a smooth curve. Let $\beta_{1}$ be a curve in $\widetilde{W}^{u}\left(\gamma_{0}\right)$ from $p_{1}$ to $g\left(p_{1}\right)$ and so that $\beta_{1}$ is smooth and transverse to $\widetilde{\Phi}$. Since $f$ and $g$ commute then $\xi=\alpha_{1} * g\left(\beta_{1}\right) *\left(f\left(\alpha_{1}\right)\right)^{-1} *\left(\beta_{1}\right)^{-1}$ is a closed loop in $\widetilde{M}$, which is transverse to $\widetilde{\Phi}$. Then $\Theta(\xi)$ is the boundary of a "parallelogram" in $\Theta(\mathcal{S})$ and it is easy to produce an embedded smooth disk $D_{1}$ in $\widetilde{M}$ which is transverse to $\widetilde{\Phi}$ and so that $\partial D_{1}=\xi$.

Since the sides of $D_{1}$ are $\beta_{1}, g\left(\beta_{1}\right), \alpha_{1}, f\left(\alpha_{1}\right)$, it follows that after a small perturbation of $D_{1}$ we may assume that $D=\pi\left(D_{1}\right)$ is a smooth, immersed closed 


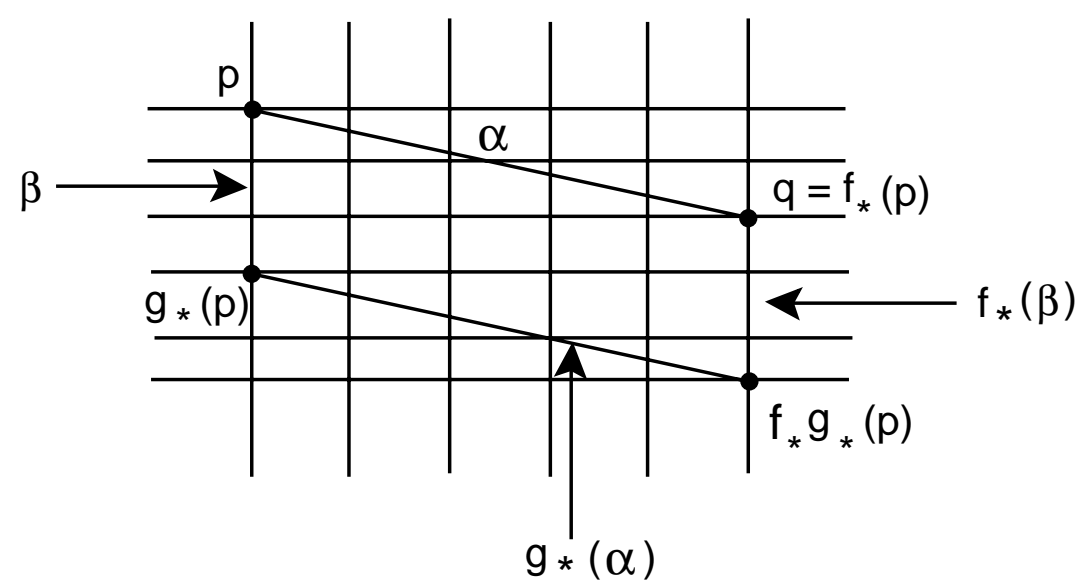

Figure 13. Producing a transverse torus.

surface transverse to $\Phi$. Using cut and paste techniques [He,Ja], as explicit done by Fried [Fr], one can eliminate all triple points of intersection and double curves of intersection, transforming $D$ into a union of embedded surfaces transverse to $\Phi$. Any such surface has induced stable and unstable foliations hence it has zero Euler characteristic. It is transverse to the flow, hence it is two sided in $M$ and as $M$ is orientable, then this transverse surface has to be a torus.

If $\Phi$ is not orientable, lift to a finite double cover $M_{2}$ of $M$ where the lifted flow is orientable. The image in $M$ of the transverse torus in $M_{2}$ is an (immersed) torus in $M$, so cut and paste techniques yield the result.

\section{Product regions, infinite branching and scalloped regions}

In this section we first show that the existence of product regions implies that the flow is $\mathbf{R}$-covered. The main difficulty is that we do not assume that $\Phi$ is transitive, in which case the proof is very simple [Fe5]. This result is used to give a detailed description of the structure induced by infinite branching in $\widetilde{\mathcal{F}}^{s}$ or $\widetilde{\mathcal{F}}^{u}$.

Theorem 5.1. Let $\Phi$ be an Anosov flow in $M^{3}$. If there is a product region in $\widetilde{M}$ then $\Phi$ is topologically conjugate to a suspension Anosov flow.

Proof. We may assume that $\Phi$ is orientable. Suppose that there is a positive unstable product region defined by $\eta \subset \widetilde{W^{s s}}\left(y_{1}\right)$. If $\Omega$ is the nonwandering set of $\Phi$, then $W^{s}(\Omega)=M[\mathrm{Pu}-\mathrm{Sh}]$, even if $\Phi$ is not transitive. As the periodic orbits are dense in $\Omega$ [Sm,Pu-Sh], there is a periodic orbit $\gamma$ of $\widetilde{\Phi}$ with $\widetilde{W^{s}}(\gamma) \cap \eta \neq \emptyset$. 


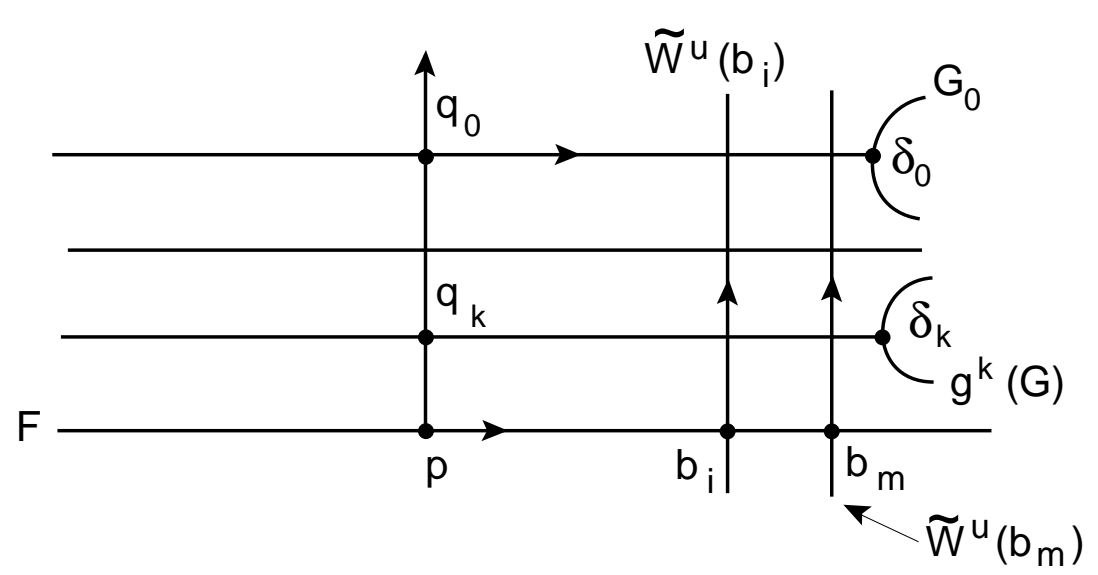

Figure 14. Boundary components of product region with infinite basis segment. $\widetilde{W}^{s}\left(b_{i}\right) \rightarrow$ $\cup_{j \in \mathbf{Z}} G_{j}$, when $i \rightarrow+\infty$.

Let $p \in \gamma$. Let $\tau$ be an open segment in $\widetilde{W}^{s s}(p)$ with $p \in \tau$, so that for any $z \in \tau, \widetilde{W}^{u}(z) \cap \eta \neq \emptyset$. Hence $\tau$ is a basis segment of a product region. Let $g$ be a generator of $\mathcal{T}\left(\widetilde{W}^{s}(p)\right)$. For any $y_{2} \in \tau, \mathcal{J}_{+}^{u}\left(y_{2}\right)=\mathcal{J}_{+}^{u}(p)$. Since $g\left(\widetilde{W}_{+}^{u}(p)\right)=$ $\widetilde{W}_{+}^{u}(p)$, then

$$
\mathcal{J}_{+}^{u}\left(g^{i}\left(y_{2}\right)\right)=g^{i}\left(\mathcal{J}_{+}^{u}\left(y_{2}\right)\right)=\mathcal{J}_{+}^{u}(p), \forall i \in \mathbf{Z}, \quad \text { hence } \mathcal{A}=\bigcup_{y_{2} \in \widetilde{W}^{s s}(p)} \widetilde{W}_{+}^{u}\left(y_{2}\right)
$$

is a product region with an infinite basis segment $\widetilde{W}^{s s}(p)$.

Claim. $\partial \mathcal{A}=\widetilde{W}^{s}(p)$.

Suppose not and let $a \in \partial \mathcal{A}-\widetilde{W}^{s}(p)$. Let $a_{i} \in \mathcal{A}$ with $a_{i} \rightarrow a$ and $b_{i}=\widetilde{W}^{u}\left(a_{i}\right) \cap$ $\widetilde{W}^{s s}(p)$. Assume $b_{i} \in \widetilde{W}_{+}^{s s}(p)$. Notice that $\widetilde{W}^{u}(a) \cap \widetilde{W}^{s}(p)=\emptyset$ and in addition $\widetilde{W}^{u}(a) \subset \partial \mathcal{A}$. If $b_{i} \nrightarrow \infty$ in $\widetilde{W}_{+}^{s s}(p)$, assume up to subsequence that $b_{i} \rightarrow b_{0}$. Since $\widetilde{W^{s}}(a) \cap \mathcal{A} \neq \emptyset$ and $\mathcal{A}$ is a product region then $\widetilde{W}^{s}(a) \cap \widetilde{W}^{u}(q) \neq \emptyset, \forall q \in \widetilde{W}^{s s}(p)$. But if $q$ is in front of $\widetilde{W}^{u}\left(b_{0}\right)$ then $\widetilde{W}^{u}(q) \cap \widetilde{W}^{s}(a)=\emptyset$, contradiction. Hence $b_{i} \rightarrow \infty$ in $\widetilde{W}_{+}^{s s}(p)$.

Let $G=\widetilde{W}^{u}(a)$ and $F=\widetilde{W^{s}}(p)$. Notice that $g^{n}(G) \neq g^{m}(G)$ for $n \neq m \in \mathbf{Z}$. Otherwise there is an orbit $\delta$ of $\widetilde{\Phi}$ in $G$ with $g(\delta)=\delta$. But $\widetilde{W}^{s}(\delta) \cap \widetilde{W}^{u}(p) \neq \emptyset$, a contradiction to both left invariant under $g$.

Since $g^{k}(\mathcal{A})=\mathcal{A}$, then $g^{k}(G) \subset \partial \mathcal{A}$. As $b_{i} \rightarrow+\infty$ in $\widetilde{W}_{+}^{s s}(p)$, then the $g^{k}(G), k \in \mathbf{Z}$ are all not separated from each other. By theorem $4.3, g^{k}(G)$ contains a periodic orbit $\delta_{k}$. Take inverse if needed so that $\widetilde{W}_{-}^{s}\left(\delta_{k}\right) \cap \widetilde{W}_{+}^{u u}(p)=q_{k}=$ $g^{k}\left(q_{0}\right) \rightarrow p$ as $k \rightarrow+\infty$, see fig. 14 . 
Let $f$ be a generator of $\mathcal{T}\left(G_{0}\right)$. Then $f\left(G_{i}\right)=G_{i}, \forall i \in \mathbf{Z}$, so $f\left(\widetilde{W}^{s}\left(\delta_{i}\right)\right)=$ $\widetilde{W^{s}}\left(\delta_{i}\right)$. As $\widetilde{W}^{s}\left(\delta_{i}\right) \rightarrow F$ as $i \rightarrow+\infty$, then $f(F)$ is not separated from $F$. The case $f(F)=F$ is ruled out as above. If $f(F) \neq F$ assume that $f\left(\widetilde{W}^{u}(p)\right)$ is in the front of $\widetilde{W}^{u}(p)$. Then $f\left(\widetilde{W}^{u}(p)\right) \cap \widetilde{W}^{u}(p)=\emptyset$, hence $f\left(\widetilde{W}^{u}(p)\right) \cap \mathcal{A}=\emptyset$, which implies that $f\left(\widetilde{W}^{u}(p)\right)$ separates $\mathcal{A}$ from all $G_{i}$. This contradicts $G_{i} \subset \partial \mathcal{A}$ and finishes the proof of the claim.

Let now $\mathcal{D}=\{$ set of positive unstable product regions $\mathcal{U}$ with base segment $\widetilde{W}^{s s}(z)$ and $\left.\mathcal{U} \cap \mathcal{A} \neq \emptyset\right\}$. The above claim shows that this set is ordered by inclusion. Let $\mathcal{V} \subset \widetilde{M}$ be the union of all $\mathcal{U} \in \mathcal{D}$. Then $\mathcal{V}$ is $\widetilde{\mathcal{F}}^{s}$ saturated. If $\mathcal{V} \neq \widetilde{M}$ let $E=\widetilde{\Phi}_{\mathbf{R}}\left(\widetilde{W}^{s s}(w)\right) \subset \partial \mathcal{V}$. Since $W^{s s}(\pi(w))$ is not compact, choose a translate $f(E)$ in the back of $E$ with $E, f(E)$ intersecting a common unstable leaf $R$. Choose $\widetilde{W}^{s s}(v)$ basis of a product region and with $v$ near enough $E$ so that $f\left(\widetilde{W}^{s s}(v)\right)$ intersects $R$ between $E$ and $f(E)$. Then $f\left(\widetilde{W}^{s s}(v)\right)$ is the basis segment of a product region intersecting $\mathcal{A}$ and not contained in $\mathcal{V}$, contradiction. Hence $\mathcal{V}=\widetilde{M}$.

Therefore for any $H \in \widetilde{\mathcal{F}}^{u}, H \cap \mathcal{A} \neq \emptyset$, hence $H \cap \widetilde{W}^{s}(p) \neq \emptyset$. This shows that $\mathcal{F}^{u}$ is R-covered. Using similar arguments one shows that any leaf of $\widetilde{\mathcal{F}}^{s}$ intersects every leaf of $\widetilde{\mathcal{F}}^{u}$ and vice versa. Theorem 2.8 of [Ba2] implies that $\Phi$ is topologically conjugate to a suspension Anosov flow. This finishes the proof.

Theorem 5.1 has applications here and also in [Fe8] and [Fe10].

We now show that infinite branching is associated to a particular type of structure, called a scalloped region in $\widetilde{M}$ (or $\mathcal{O}$ ).

Theorem 5.2. Let $\Phi$ be an Anosov flow in $M^{3}$. If there is infinite branching in $\widetilde{\mathcal{F}}^{s}$, then there is associated infinite branching in $\widetilde{\mathcal{F}}^{u}$.

Proof. We use the notation from theorem 4.9. Let $\mathcal{E}=\left\{E_{i}\right\}_{i \in \mathbf{Z}} \subset \widetilde{\mathcal{F}}^{s}$ be an ordered collection of leaves non separated on their negative sides. Let $\left\{\mathcal{B}_{i}\right\}, i \in \mathbf{Z}$ be the lozenges associated to $\mathcal{E}$ and let $F_{i}$ be the leaves on the negative sides of $\mathcal{B}_{i}$. Let $\gamma_{i}$ and $\zeta_{i}$ be the periodic orbits in $E_{i}$ and $F_{i}$ respectively. Let

$$
\mathcal{S}=\bigcup_{i \in \mathbf{Z}}\left(\mathcal{B}_{i} \cup \widetilde{W}_{-}^{u}\left(\gamma_{i}\right) \cup \widetilde{W}_{+}^{u}\left(\zeta_{i}\right)\right)
$$

Recall also that all of the following sets are equal $\mathcal{J}_{-}^{u}\left(\gamma_{i}\right), i \in \mathbf{Z}, \mathcal{J}_{+}^{u}\left(\zeta_{j}\right), j \in \mathbf{Z}$.

Let $\mathcal{C}_{i}$ be the back of $\widetilde{W}^{u}\left(\gamma_{i}\right)$ and let $\mathcal{C}=\cup_{i \in \mathbf{N}} \mathcal{C}_{i}$. For any $p, q \in \widetilde{W}_{-}^{u}\left(\gamma_{0}\right)$ and any $i>0, \widetilde{W}^{u}\left(\gamma_{i}\right) \in \mathcal{J}_{+}^{s}(p) \cap \mathcal{J}_{+}^{s}(q)$. If $\mathcal{C}=\widetilde{M}$, then the intersections of $\widetilde{W}^{u}\left(\gamma_{i}\right)$ with $\widetilde{W}_{+}^{s s}(p)$ and $\widetilde{W}_{+}^{s s}(q)$ are escaping to infinity in these leaves. Hence $\mathcal{J}_{+}^{s}(p)=\mathcal{J}_{+}^{s}(q)$ and $\widetilde{W}_{-}^{u u}\left(\gamma_{0}\right)$ is the basis segment of a positive stable product region in $\widetilde{M}$. By theorem 5.1, $\Phi$ would be $\mathbf{R}$-covered contrary to hypothesis. Hence $\mathcal{C} \neq \widetilde{M}$. This is the key fact which will show that $\mathcal{S}$ has the form of a scalloped region, see fig. 15 . 


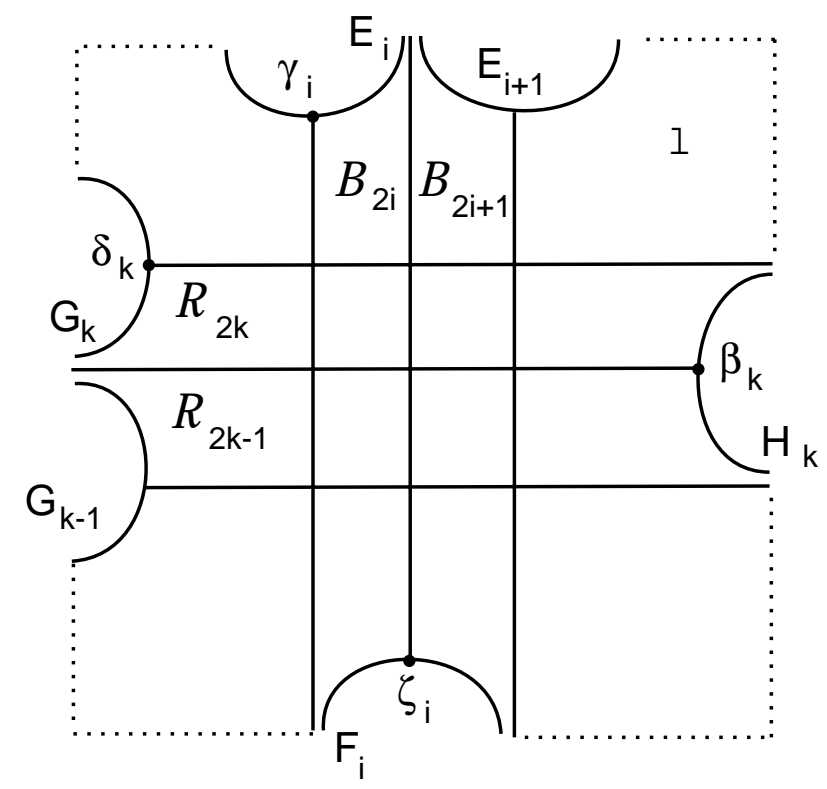

Figure 15. A scalloped region in the universal cover.

Let then $p \in \partial \mathcal{C}$, hence $\widetilde{W}^{u}(p) \subset \partial \mathcal{C}$. For $i$ big enough $\widetilde{W}_{-}^{s}(p) \cap \widetilde{W}^{u}\left(\gamma_{i}\right) \neq \emptyset$, hence $\widetilde{W}_{-}^{s}(p) \cap \widetilde{W}_{-}^{u}\left(\gamma_{i}\right) \neq \emptyset, \forall i \in \mathbf{Z}$. As a result $\widetilde{W}^{u}(p) \subset \partial \mathcal{S}$.

Since $g(\mathcal{C})=\mathcal{C}$, then $g^{n}\left(\widetilde{W}^{u}(p)\right) \subset \partial \mathcal{S}$ for any $n \in \mathbf{Z}$. If $g^{n}\left(\widetilde{W}^{u}(p)\right)=\widetilde{W}^{u}(p)$ for some $n \neq 0$, let $\beta$ be the periodic orbit in $\widetilde{W}^{u}(p)$. Then

$$
g^{n}\left(\widetilde{W}^{s}(\beta)\right)=\widetilde{W}^{s}(\beta), \quad g^{n}\left(\widetilde{W}^{u}\left(\gamma_{i}\right)\right)=\widetilde{W}^{u}\left(\gamma_{i}\right) \text { and } \widetilde{W}^{s}(\beta) \cap \widetilde{W}^{u}\left(\gamma_{i}\right) \neq \emptyset
$$

contradiction. Hence the leaves $g^{n}\left(\widetilde{W}^{u}(p)\right), n \in \mathbf{Z}$ are all distinct and all non separated from each other on their negative sides. By theorem $4.3, g^{n}\left(\widetilde{W}^{u}(p)\right)$ are all periodic and let $h$ be the indivisible covering translation leaving all invariant and acting as an expansion in the set of orbits in $\widetilde{W}^{u}(p)$.

Notice that $g\left(\widetilde{W}^{s}(p)\right)$ is in front of $\widetilde{W}^{s}(p)$. Let $H_{0}=\widetilde{W}^{u}(p), H_{1}, \ldots, H_{n}=$ $g\left(\widetilde{W}^{u}(p)\right)$ be the chain of non separated leaves from $\widetilde{W}^{u}(p)$ to $g\left(\widetilde{W}^{u}(p)\right)$. Then one constructs $\left\{H_{k}\right\}_{k \in \mathbf{Z}}$, all in $\partial \mathcal{S}$. Let $\beta_{k}$ be the periodic orbits in $H_{k}$. Then $\beta_{k}$ is the corner of two lozenges $\mathcal{R}_{2 k-1}$ and $\mathcal{R}_{2 k}$ and all $\mathcal{R}_{k}$ intersect a common unstable leaf.

Furthermore if $q \in \partial \mathcal{C}$, then $\widetilde{W}^{u}(q)$ is not separated from $H_{0}$, so $\widetilde{W}^{u}(q)$ is one of $H_{k}$. Let $\left\{G_{k}\right\}_{k \in \mathbf{Z}}$ be the sequence of leaves which form the negative unstable boundary of the lozenges $\left\{\mathcal{R}_{k}\right\}_{k \in \mathbf{Z}}$. Then $h\left(G_{k}\right)=G_{k}$ for all $k$. Let $\delta_{k}$ be the 
periodic orbit in $G_{k}$ and let

$$
\mathcal{M}=\bigcup_{k \in \mathbf{Z}}\left(\mathcal{R}_{k} \cup \widetilde{W}_{-}^{s}\left(\beta_{k}\right) \cup \widetilde{W}_{+}^{s}\left(\delta_{k}\right)\right)
$$

Given $l \in \mathbf{Z}$ then for $j>0$ big enough $\widetilde{W}_{-}^{u}\left(\gamma_{j}\right) \cap \widetilde{W}_{-}^{s}\left(\beta_{l}\right) \neq \emptyset$. Since all $\mathcal{J}_{-}^{s}\left(\beta_{k}\right), k \in \mathbf{Z}$ are all equal as are all $\mathcal{J}_{-}^{u}\left(\gamma_{i}\right)$ this implies that for any $i, k \in$ $\mathbf{Z}, \mathcal{B}_{i} \cap \mathcal{R}_{k} \neq \emptyset$. As $g\left(\mathcal{B}_{i}\right)=\mathcal{B}_{i}$ for any $i \in \mathbf{Z}$ and there is $n_{0} \in \mathbf{Z}$ so that $g\left(\mathcal{R}_{k}\right)=\mathcal{R}_{k+n_{0}}$ for any $k \in \mathbf{Z}$, then for any $i \in \mathbf{Z}, \mathcal{B}_{i} \subset \mathcal{M}$. In the same way $\widetilde{W}_{-}^{u}\left(\gamma_{i}\right), \widetilde{W}_{+}^{u}\left(\beta_{i}\right) \subset \mathcal{M}$.

In addition notice that $g^{m}\left(\widetilde{W}^{s}\left(\beta_{0}\right)\right) \rightarrow \cup_{i \in \mathbf{Z}} E_{i}=\mathcal{E}$ as $m \rightarrow+\infty$. As $f\left(\widetilde{W}^{s}\left(\beta_{k}\right)\right)$ $=\widetilde{W}^{s}\left(\beta_{k}\right), \forall k \in \mathbf{Z}$ then $f$ leaves invariant the set $\mathcal{E}$. Therefore there is $j_{0} \in \mathbf{N}$ so that $f\left(E_{i}\right)=E_{i+j_{0}}$ for all $i \in \mathbf{Z}$. Since $f\left(\mathcal{R}_{k}\right)=\mathcal{R}_{k}, \forall k \in \mathbf{Z}$, then the same argument as above implies that $\mathcal{R}_{k} \subset \mathcal{S}$, for any $k \in \mathbf{Z}$. The important conclusion is that $\mathcal{S}=\mathcal{M}$. The region $\mathcal{S}$ is called a scalloped region, see fig. 15. The region is decomposed into two essentially different ways as an infinite union of disjoint lozenges (plus some of the sides). Whenever there is infinite branching in one of $\widetilde{\mathcal{F}}^{s}$ or $\widetilde{\mathcal{F}}^{u}$, there is an associated scalloped region.

\section{Examples}

As explained before the non $\mathbf{R}$-covered property can occur for transitive and intransitive Anosov flows. We now consider infinite and finite branching.

The Anosov flow constructed by Bonatti and Langevin [Bo-La] is transitive and has infinite branching. The scalloped region of thisflow was explained in detail in [Ba3]. The Bonatti-Langevin flow is the simplest Anosov flow with infinite branching in the sense that there is only one orbit $\nu$ of $\Phi$ which does not intersect the transverse torus constructed by the infinite branching. In this case all the periodic orbits in the boundary of the scalloped region are lifts of $\nu$.

As for examples of finite branching we will produce in the next section a large family of non $\mathbf{R}$-covered Anosov flows in hyperbolic manifolds. These are transitive and by corollary 4.8 they cannot have infinite branching. Hence they have only finite branching.

As for intransitive examples with finite branching we consider the flows constructed by Franks and Williams [Fr-Wi]. The structure of $\widetilde{\mathcal{F}}^{s}$ near a branching leaf is described in fig. 16 . Notice that $F_{1}$ is not separated from $F_{2}$ on their negative sides, $F_{2}$ not separated from $F_{3}$ on their positive sides and so on. In fact the only non separated leaf from $F_{1}$ in the negative side of $F_{1}$ is $F_{2}$. Hence there is only finite branching and $\mathcal{E}_{b}\left(F_{i}\right)$ has always two elements. 


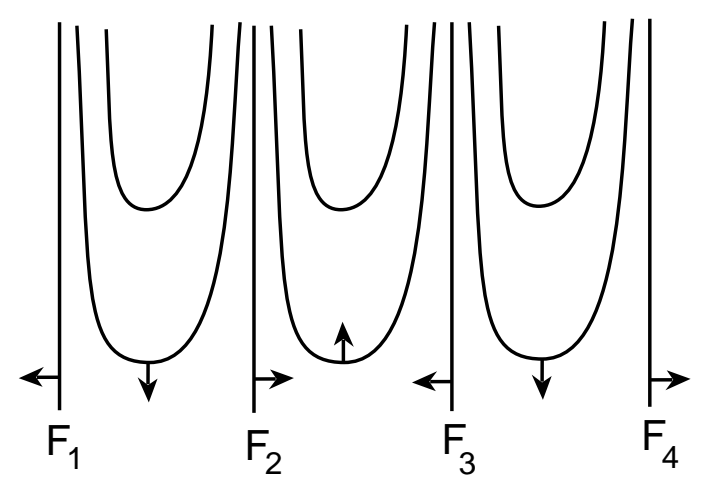

Figure 16. The structure of non separated leaves of $\widetilde{\mathcal{F}}^{s}$ in the Franks-Williams example. Arrows indicate positive transversal orientation to $\widetilde{\mathcal{F}}^{s}$.

\section{Non R-covered Anosov flows in hyperbolic 3-manifolds}

In this section we produce examples of transitive, non $\mathbf{R}$-covered Anosov flows in hyperbolic 3-manifolds. Recall that any Anosov flow in a hyperbolic 3-manifold is necessarily transitive [Fe4].

Theorem 6.1. There is a large class of transitive, non $\mathbf{R}$-covered Anosov flows in hyperbolic 3-manifolds, including all Anosov flows in non orientable hyperbolic 3-manifolds.

Proof. Theorem $C$ of [Ba2] states that if $\Phi$ is an $\mathbf{R}$-covered Anosov flow in $M^{3}$, then either $\Phi$ is topologically conjugate to a suspension Anosov flow or the underlying manifold is orientable (notice that Barbot uses the term "product" instead of Rcovered). Since hyperbolic manifolds can never be the underlying manifolds of suspension Anosov flows, it suffices to produce Anosov flows in non orientable hyperbolic 3-manifolds.

Consider therefore the suspension of an orientation reversing Anosov diffeomorphism of the torus $T^{2}$. Let $M$ be the underlying manifold of the suspension and let $\alpha$ be an orientation preserving closed orbit of the flow. As described by Goodman [Go] and Fried [Fr], $(n, 1)$ Dehn surgery along $\alpha$ yields an Anosov flow in the surgered manifold $M_{(n, 1)}$.

It is well known that $(M-\alpha)$ is irreducible, atoroidal and homeomorphic to the interior of a compact 3-manifold with boundary [Th1]. By Thurston's hyperbolization theorem [Th2,Mor] it follows that $(M-\alpha)$ admits a complete hyperbolic structure of finite volume. By the hyperbolic Dehn surgery theorem [Th1], most Dehn fillings on $(M-\alpha)$ yield closed, hyperbolic manifolds. Since $M$ was non orientable, all of these manifolds are non orientable. Whenever the Dehn surgery coefficient is of the form $(n, 1)$, the surgered manifold admits an Anosov 


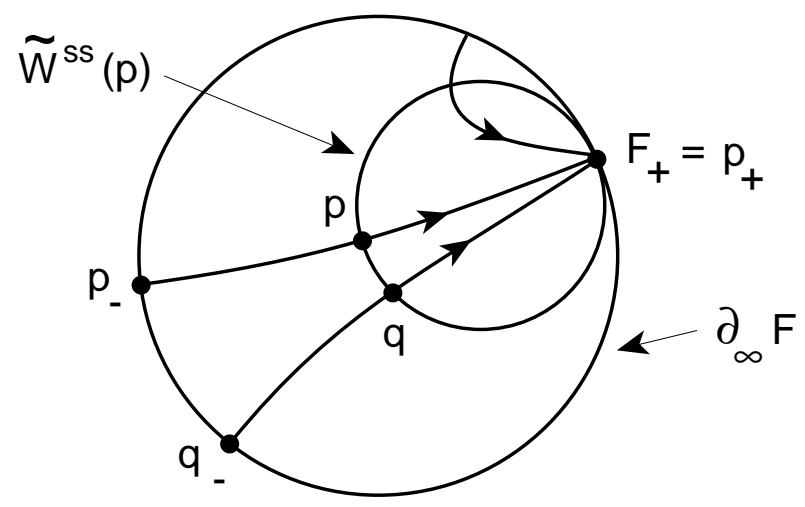

Figure 17. Intrinsic ideal points.

flow. This produces a large class of Anosov flows in non orientable hyperbolic 3-manifolds and finishes the proof.

\section{Continuous extension of Anosov foliations}

In this section we study the asymptotic behavior of leaves of $\widetilde{\mathcal{F}}^{s}$ and $\tilde{\mathcal{F}}^{u}$ when $\pi_{1}(M)$ is negatively curved.

If $\Phi$ is an Anosov flow in $M^{3}$, Sullivan [Su] showed that the intrinsic geometry of leaves of $\widetilde{\mathcal{F}}^{s}$ and $\widetilde{\mathcal{F}}^{u}$ is negatively curved in the large as defined by Gromov [Gr]. Then any leaf $F \in \widetilde{\mathcal{F}}^{s} \cup \widetilde{\mathcal{F}}^{u}$ has a canonical compactification with an intrinsic ideal boundary $\partial_{\infty} F[\mathrm{Gr}]$ and $\partial_{\infty} F$ is always homeomorphic to a circle [Fe2]. All of this works without any assumption on $M$ or $\Phi$.

If $F \in \widetilde{\mathcal{F}}^{s}$ then the intrinsic ideal points are the (distinct) negative limit points of flow lines in $F$ and the common positive limit point of all flow lines [Fe3]. The intrinsic geometry of $F \in \widetilde{\mathcal{F}}^{s}$ is similar to the hyperbolic plane $\mathbf{H}^{2}$ where the flow lines correspond to the geodesics in $\mathbf{H}^{2}$ which have a common limit point in the ideal boundary of $\mathbf{H}^{2}$, see fig. 17 .

If $p \in F \in \widetilde{\mathcal{F}}^{s}$, let $p_{-}$be the intrinsic negative ideal point of the flow line through $p$, that is,

$$
p_{-}=\lim _{t \rightarrow-\infty} \widetilde{\Phi}_{t}(p) \in \partial_{\infty} F,
$$

where the limit is taken in $F \cup \partial_{\infty} F$, see fig. 17. Similarly define $p_{+}$. For any $p, q \in F \in \widetilde{\mathcal{F}}^{s}, p_{+}=q_{+} \in \partial_{\infty} F$ and this is also denoted by $F_{+}$. Furthermore if $p_{i} \in \widetilde{W}^{s s}(p)$ and $p_{i} \rightarrow \infty$ in $\widetilde{W}^{s s}(p)$, then $\left(p_{i}\right)_{-} \rightarrow p_{+}$as points in $\partial_{\infty} F[\mathrm{Fe} 3]$. 
This can be clearly seen in the model of $\mathbf{H}^{2}$. If $p, q$ are in the same flow line $\alpha$ of $\widetilde{\Phi}$, then $p_{-}=q_{-}$, which is also denoted by $(\alpha)_{-} \in \partial_{\infty} F$. Similarly $(\alpha)_{+}=F_{+}$.

From now on we assume that $\pi_{1}\left(M^{3}\right)$ is negatively curved as defined by Gromov [Gr], who constructed a canonical compactification of $\widetilde{M}$ with an ideal boundary $\partial \widetilde{M}$. Since $M$ is irreducible [Ro], Bestvina and Mess [Be-Me] showed that $\partial \widetilde{M}$ is homeomorphic to a sphere, denoted by $S_{\infty}^{2}$. Furthermore $\widetilde{M} \cup S_{\infty}^{2}$ is homeomorphic to a closed 3-ball.

We say that $\widetilde{\Phi}$ has the continuous extension property if for any leaf $F \in \widetilde{\mathcal{F}}^{s} \cup \widetilde{\mathcal{F}}^{u}$, the embedding $\varphi_{F}: F \rightarrow \widetilde{M}$, extends continuously to $\varphi_{F}: F \cup \partial_{\infty} F \rightarrow \widetilde{M} \cup S_{\infty}^{2}$. This gives a continuous parametrization of the limit sets $\Lambda_{F}=\varphi_{F}\left(\partial_{\infty} F\right)$. This also implies that there is a continuous function

$$
\eta_{-}: \widetilde{M} \rightarrow S_{\infty}^{2}, \quad \eta_{-}(x)=\lim _{t \rightarrow-\infty} \widetilde{\Phi}_{t}(x)
$$

where the limit is computed in $\widetilde{M} \cup S_{\infty}^{2}$. The function $\eta_{-}$is constant along an orbit $\alpha$ of $\widetilde{\Phi}$, with value $\eta_{-}(\alpha)=\varphi_{\widetilde{W}^{s}(\alpha)}\left(\alpha_{-}\right)$. Furthermore for any $G \in \widetilde{\mathcal{F}}^{u}, \eta_{-}$ is a constant function in $G$ with value $\varphi_{G}\left(G_{-}\right)$. Similarly define $\eta_{+}: \widetilde{M} \rightarrow S_{\infty}^{2}$. Given any set

$$
X \subset \widetilde{M}, \text { let } \eta_{-}(X)=\bigcup_{x \in X} \eta_{-}(x)
$$

The continuous extension property implies that for any $F \in \widetilde{\mathcal{F}}^{s}$ and any $p \in F$, then

$$
\Lambda_{F}=\varphi_{F}\left(\partial_{\infty} F\right)=\eta_{-}\left(\widetilde{W}^{s s}(p)\right) \cup \eta_{+}(p) .
$$

In [Fe6] we study the continuous extension property for $\mathbf{R}$-covered flows.

Recall that the limit set of a subset $B$ of $\widetilde{M}$ is $\Lambda_{B}=\bar{B} \cap S_{\infty}^{2}$, where the closure is taken in $\widetilde{M} \cup S_{\infty}^{2}$. Also recall that $\widetilde{\mathcal{F}}^{s}, \widetilde{\mathcal{F}}^{u}$ are transversely oriented. Given $F \in \widetilde{\mathcal{F}}^{s}$ or $\widetilde{\mathcal{F}}^{u}$ and $p \in S_{\infty}^{2}-\Lambda_{F}$, we say that $p$ is above $F$ if there is a neighborhood $U$ of $p$ in $\widetilde{M} \cup S_{\infty}^{2}$ so that $U \cap \widetilde{M}$ is in front of $F$. Otherwise we say that $p$ is below $F$. Given a connected component of $S_{\infty}^{2}-\Lambda_{F}$ either all of its points are above $F$ and we say this component is above $F$, otherwise we say the component is below $F$.

Theorem 7.1. Let $\Phi$ be a non $\mathbf{R}$-covered Anosov flow in $M^{3}$ with negatively curved $\pi_{1}(M)$. Suppose that $\widetilde{\Phi}$ has the continuous extension property. Then for any leaf $C \in \widetilde{\mathcal{F}}^{s} \cup \widetilde{\mathcal{F}}^{u}$, the limit set $\Lambda_{C}$ is a Sierpinski curve, that is the complement of a countable, dense union of open disks in the sphere $S_{\infty}^{2}$. In addition if $M$ is hyperbolic, then there is $k<2$ so that the union of all limit sets,

$$
\mathcal{N}=\bigcup_{F \in \widetilde{\mathcal{F}}^{s} \cup \widetilde{\mathcal{F}}^{u}} \Lambda_{F}
$$




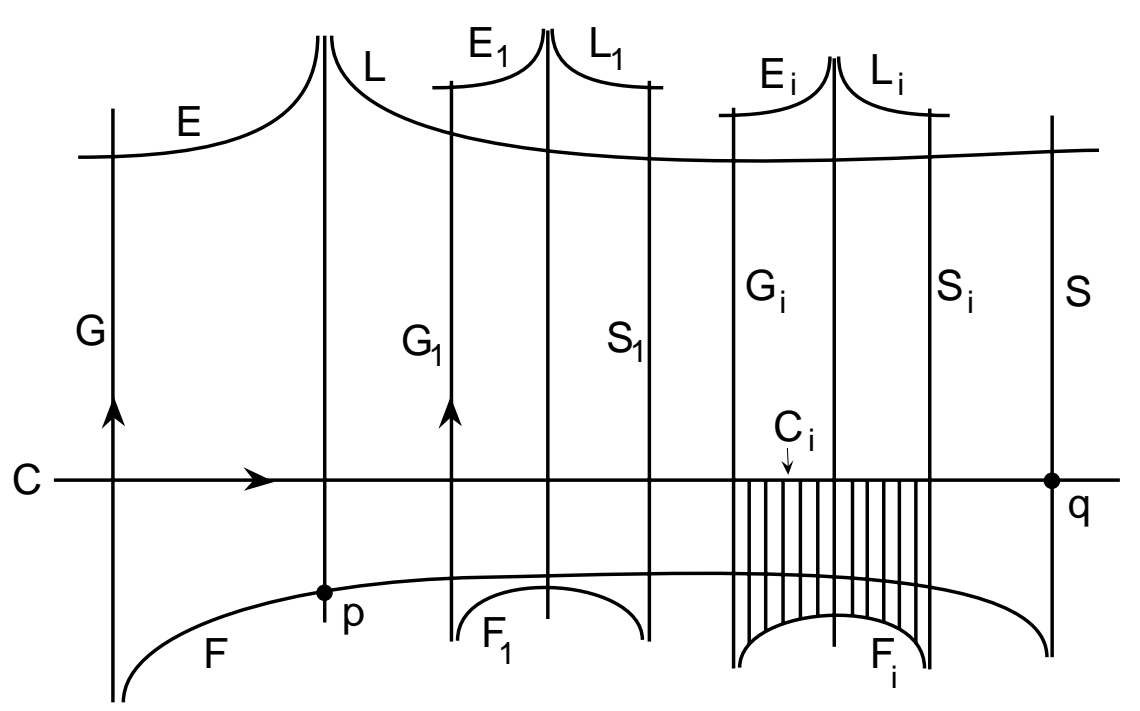

Figure 18. Sequence of lozenges.

has Hausdorff dimension $<k$. In particular every limit set $\Lambda_{F}$ has zero Lebesgue measure in the sphere at infinity.

Proof. We first prove that $\Lambda_{C} \neq S_{\infty}^{2}$ and then use part of this proof to show that limit sets of leaves are Sierpinski curves. The first part is similar to the proof of theorem 5.5 of [Fe4], except that in [Fe4] we assumed the very strong hypothesis of quasigeodesic behavior of $\Phi$, which is not assumed here. The continuous extension property is a much weaker property than quasigeodesic behavior.

Since $\Phi$ is transitive, $\widetilde{\mathcal{F}}^{s}$ has branching in the positive and negative directions [Fe5]. Using theorem 4.7 we produce $\Delta$, a union of two adjacent lozenges in $\widetilde{M}$ and a common side, all intersecting a common stable leaf so that: (1) the boundary of $\Delta$ has unstable sides in $G, S \in \widetilde{\mathcal{F}}^{u}$, and stable sides in $E, F, L \in \widetilde{\mathcal{F}}^{s}(2) E, L$ are not separated on their negative sides, (3) $G$ is in the back of $S$ and (4) $E \cap G \neq \emptyset$, $L \cap S \neq \emptyset$, see fig. 18. By $G$ we mean the half leaf in the boundary of $\Delta$. Then $\pi(G)$ is dense in $M$ [Fe3].

Let $C \in \widetilde{\mathcal{F}}^{s}$ be a leaf intersecting both $G$ and $S$, hence $C$ intersects $\Delta$. Choose a covering translation $g_{1}$ so that

$$
g_{1}(G) \cap F \neq \emptyset, \quad g_{1}(G) \cap L \neq \emptyset .
$$

As $g_{1}(F), g_{1}(G)$ form a perfect fit, then $g_{1}(F)$ is in the back of $F$. As $g_{1}(L), g_{1}(E)$ form a perfect fit, then both are in the front of $L$. Since $g_{1}(S) \cap g_{1}(L) \neq \emptyset$ and $g_{1}(S), g_{1}(F)$ form a perfect fit, it follows that $g_{1}(S)$ is in the front of $g_{1}(G)$, in the back of $S$ and intersects both $L$ and $F$. Inductively choose covering translations 
$g_{i}$ so that $g_{i}(G)$ is in the back of $S$,

$$
g_{i}(G) \cap F \neq \emptyset, \quad g_{i}(G) \cap L \neq \emptyset, \quad g_{i}(G) \rightarrow S \text { as } i \rightarrow \infty,
$$

and $g_{i}(G)$ is in the front of $g_{i-1}(S)$, see fig. 18. Let $G_{i}=g_{i}(G)$ and similarly define $F_{i}, L_{i}, S_{i}$ and $E_{i}$.

Let $C_{i}=C \cap g_{i}(\Delta)$. For any flow line $\gamma \in F_{i}, \widetilde{W}^{u}(\gamma)$ intersects $C_{i}$ and vice versa. Hence $\eta_{-}\left(C_{i}\right)=\eta_{-}\left(F_{i}\right)$. Let $q \in C \cap S$. By continuity of $\eta_{-}$, there is a neighborhood $Y$ of $q$ in $\widetilde{M}$ so that $\eta_{-}(Y)$ is contained in a small neighborhood $Y^{\prime}$ of $\eta_{-}(q)$ in $S_{\infty}^{2}$. As $C_{i} \cap \widetilde{W}^{s s}(q) \rightarrow q$, then $\eta_{-}\left(C_{i}\right) \subset Y^{\prime}$ for $i$ big enough. Therefore $\eta_{-}\left(F_{i}\right) \subset Y^{\prime}$ and as a result $\Lambda_{F_{i}}$ is contained in the closure of $Y^{\prime}$ and is not $S_{\infty}^{2}$. This shows that $\Lambda_{F}=\Lambda_{g_{i}^{-1}\left(F_{i}\right)}=g_{i}^{-1}\left(\Lambda_{F_{i}}\right) \neq S_{\infty}^{2}$.

Since $\widetilde{\mathcal{F}}^{s}$ has branching in the positive and negative directions and $\Lambda_{F} \neq S_{\infty}^{2}$, then theorem 3.3 of [Fe9] shows that, for any $L^{\prime} \in \widetilde{\mathcal{F}}^{s}$, there are components of $S_{\infty}^{2}-\Lambda_{L^{\prime}}$ above $L^{\prime}$ and components of $S_{\infty}^{2}-\Lambda_{L^{\prime}}$ below $L^{\prime}$. For each $i$ let $Z_{i}$ be a component of $S_{\infty}^{2}-\Lambda_{F_{i}}$ below $F_{i}$. Since $C$ is in front of $F_{i}, Z_{i} \cap \Lambda_{C}=\emptyset$. Hence $Z_{i}$ is contained in a component $Z_{i}^{*}$ of $S_{\infty}^{2}-\Lambda_{C}$ which is below $C$. The argument above used to prove that $\Lambda_{F_{i}} \neq S_{\infty}^{2}$ shows that $\Lambda_{F_{i}} \subset \Lambda_{C}$, hence the component $Z_{i}^{*}$ of $S_{\infty}^{2}-\Lambda_{C}$ is equal to $Z_{i}$.

For each $i, Z_{i}$ is below $F_{i}$. In addition for each $i \neq j, F_{i}$ is in the front of $F_{j}$ and $F_{j}$ is in the front $F_{i}$. This implies that $Z_{i} \cap Z_{j}=\emptyset$. Hence $\left\{Z_{i}\right\}, i \in \mathbf{N}$ is an infinite family of distinct components of $S_{\infty}^{2}-\Lambda_{C}$ below $C$. Using branching of $\widetilde{\mathcal{F}}^{s}$ in the negative direction, one constructs countably many components of $S_{\infty}^{2}-\Lambda_{C}$ above $C$.

Since $\Phi$ is transitive, then for any $C^{\prime} \in \widetilde{\mathcal{F}}^{s}$ there is a covering translation $f$ so that $f\left(C^{\prime}\right) \cap \Delta \neq \emptyset$. The argument above shows that $S_{\infty}^{2}-\Lambda_{f\left(C^{\prime}\right)}$ has infinitely many components above and below $f\left(C^{\prime}\right)$. Translation by $f^{-1}$ yields the same result for $C^{\prime}$. By theorem 4.4 of [Fe9], $\Lambda_{C^{\prime}}$ has empty interior, hence $\Lambda_{C^{\prime}}$ is a Sierpinski curve.

Suppose now that $M$ is hyperbolic. Again since $\widetilde{\mathcal{F}}^{s}, \widetilde{\mathcal{F}}^{u}$ have branching in the positive and negative directions, corollary 3.9 of [Fe9] shows that there is $k<2$ so that for any $F \in \widetilde{\mathcal{F}}^{s}$ or $\widetilde{\mathcal{F}}^{u}$, the Hausdorff dimension of $\Lambda_{F}$ is $<k$. In particular $\Lambda_{F}$ has zero Lebesgue measure.

Let $\left\{W_{i}\right\}_{i \in \mathbf{N}}$, be a collection of 2-dimensional disks in $\widetilde{M}$ transverse to $\widetilde{\Phi}$, so that each $W_{i}$ projects to a rectangle in $\mathcal{O}$ and so that any flow line of $\widetilde{\Phi}$ intersects at least one of the $W_{i}$. Then

$$
\mathcal{N}=\bigcup_{F \in \widetilde{\mathcal{F}}^{s} \cup \widetilde{\mathcal{F}}^{u}} \Lambda_{F}=\bigcup_{z \in \widetilde{M}}\left(\eta_{-}(z) \cup \eta_{+}(z)\right)=\bigcup_{i \in \mathbf{N}}\left(\eta_{-}\left(W_{i}\right) \cup \eta_{+}\left(W_{i}\right)\right) .
$$

Let $F_{i}$ be a stable leaf intersecting $W_{i}$. Since $W_{i}$ projects to a rectangle in $\mathcal{O}$ then $\eta_{-}\left(W_{i}\right)=\eta_{-}\left(W_{i} \cap F_{i}\right) \subset \Lambda_{F_{i}}$. Since $\Lambda_{F_{i}}$ has Hausdorff dimension less than $k$ and $\mathcal{N}$ is a countable union of such sets, then $\mathcal{N}$ also has Hausdorff dimension $<k$. This finishes the proof. 


\section{References}

[An] D. V. Anosov, Geodesic flows on closed Riemannian manifolds with negative curvature, Proc. Steklov Inst. Math. 90 (1969).

[An-Si] D. V. Anosov and Y. Sinai, Some smoothly ergodic systems, Russian Math. Surveys 22 5 (1967), 103-167.

[Ba1] T. Barbot, Géométrie transverse des flots d'Anosov, Thesis, École Norm. Sup., Lyon, 1992.

[Ba2] T. Barbot, Caractérisation des flots d'Anosov en dimension 3 par leurs feuilletages faibles, Erg. Th. Dyn. Sys. 15 (1995), 247-270.

[Ba3] T. Barbot, Mise en position optimale d'un tore par rapport à un flot d'Anosov, Comm. Math. Helv. 70 (1995), 113-160.

[Ba4] T. Barbot, Flots d'Anosov sur les variétés graphées au sens de Waldhausen, Ann. Inst. Fourier Grenoble 46 (1996), 1451-1517.

[Be-Me] M. Bestvina and G. Mess, The boundary of negatively curved groups, Jour. Amer. Math. Soc. 4 (1991), 469-481.

[Bon] F. Bonahon, Bouts des variétés hyperboliques de dimension 3, Ann. of Math. 124 (1986), $71-158$.

[Bo-La] C. Bonatti and R. Langevin, Un example de flot D'Anosov transitif transverse a un tore et non conjugue a une suspension, Erg. Th. Dyn. Sys. 14 (1994), 633-643.

[Bow] R. Bowen, Equilibrium states and the ergodic theory of Anosov diffeomorphims, Lecture Notes in Mathematics 470, Springer Verlag, 1975.

[Br] M. Brunella, On the discrete Godbillon-Vey invariant and Dehn surgery on geodesic flows, preprint, 1994.

[Ca-Th] J. Cannon and W. Thurston, Group invariant Peano curves, to appear.

[Ch] J. Christy, Intransitive Anosov flows on 3-manifolds, to appear in C.B.M.S. lecture series.

[Fe1] S. Fenley, Asymptotic properties of depth one foliations in hyperbolic 3-manifolds, Jour. Diff. Geom. 36 (1992), 269-313.

[Fe2] S. Fenley, Quasi-isometric foliations, Topology 31 (1992), 667-676.

[Fe3] S. Fenley, Anosov flows in 3-manifolds, Ann. of Math. 139 (1994), 79-115.

[Fe4] S. Fenley, Quasigeodesic Anosov flows and homotopic properties of closed orbits, Jour. Diff. Geo. 41 (1995), 479-514.

[Fe5] S. Fenley, One sided branching in Anosov foliations, Comm. Math. Helv. 70 (1995), 248-266.

[Fe6] S. Fenley, Continuous extension of Anosov foliations in 3-manifolds with negatively curved fundamental group, to appear in Pac. Jour. Math.

[Fe7] S. Fenley, Homotopic indivisibility of closed orbits of Anosov flows, Math. Zeit 225 (1997), 289-294.

[Fe8] S. Fenley, Incompressible tori transverse to Anosov flows in 3-manifolds, Erg. Th. Dyn. Sys. 17 (1997), 105-121.

[Fe9] S. Fenley, Limit sets of foliations in hyperbolic 3-manifolds, Topology 37 (1998), 875-894.

[Fe10] S. Fenley, Foliations with good geometry, preprint, 1997.

[Fe-Mo] S. Fenley and L. Mosher, Quasigeodesic flows in hyperbolic 3-manifolds, to appear.

[Fr-Wi] J. Franks and R. Williams, Anomalous Anosov flows, Global theory of Dyn. Systems, Lecture Notes in Math. 819, Springer, 1980.

[Fr] D. Fried, Transitive Anosov flows and pseudo-Anosov maps, Topology 22 (1983), 299-303.

[Ga] D. Gabai, Convergence groups are Fuchsian groups, Ann. of Math. 136 (1992), 447-510.

[Ga-Oe] D. Gabai and U. Oertel, Essential laminations and 3-manifolds, Ann. of Math. 130 (1989), 41-73.

[Gh] E. Ghys, Flots d'Anosov sur les 3-variétés fibrés en cercles, Ergod. Th. Dyn. Sys. 4 (1984), 67-80. 
[Go] S. Goodman, Dehn surgery and Anosov flows, in Proceedings of the geometric dynamics conference, Lecture Notes in Mathematics 1007, Springer, 1983.

[Gr] M. Gromov, Hyperbolic groups. In: Essays on group theory, 75-263, Springer, 1987.

[He] J. Hempel, 3-manifolds, Ann. of Math. Studies 86, Princeton University Press, 1976.

[Im] H. Imanishi, On the theorem of Denjoy-Sacksteder for codimension one foliations without holonomy, J. Math. Kyoto U. 14 (1974), 607-634.

[Ja] W. Jaco, Lectures on 3-manifold topology, C.B.M.S. lecture series 43 (1980).

[Ja-Sh] W. Jaco and P. Shalen, Seifert fibered spaces in 3-manifolds, Memoirs of the A. M. S. 21 (220) (1979).

[Jo] K. Johannson, Homotopy equivalences of 3-manifolds with boundaries, Lecture Notes in Math. 761, Springer, 1979.

[Mor] J. Morgan, On Thurston's uniformization theorem for 3-dimensional manifolds. In: The Smith Conjecture, J. Morgan and H. Bass, eds., Academic Press, New York, 1984, pp. $37-125$.

[Mos1] L. Mosher, Examples of quasigeodesic flows on hyperbolic 3-manifolds. In: Proceedings of the Ohio State University Research Semester on Low dimensional topology, W. de Gruyter, 1992.

[Mos2] L. Mosher, Dynamical systems and the homology norm of a 3-manifold, I. Efficient intersection of surfaces and flows, Duke Math. Jour. 65 (1992), 449-500.

[No] S. P. Novikov, Topology of foliations, Trans. Moscow Math. Soc. 14 (1963), 268-305.

[Pa] F. Palmeira, Open manifolds foliated by planes, Ann. of Math. 107 (1978), 109-131.

[P11] J. Plante, Anosov flows, Amer. Jour. Math. 94 (1972), 729-754.

[P12] J. Plante, Anosov flows, transversely affine foliations and a conjecture of Verjovsky, $J$. London Math. Soc. 23 (2) (1981), 359-362.

[P13] J. Plante, Solvable groups acting on the line, Trans. Amer. Math. Soc. 278 (1983), 401-414.

[Pu-Sh] C. Pugh and M. Shub, The $\Omega$-stability theorem for flows, Invent. Math. 11 (1970), $150-158$.

[Ro] H. Rosenberg, Foliations by planes, Topology 7 (1968), 131-138.

[Sh] M. Shub, Global stability of dynamical systems, Springer Verlag, 1987.

[Sm] S. Smale, Differentiable dynamical systems, Bull. Amer. Math. Soc. 73 (1967), 747817.

[So] V. V. Solodov, On the universal cover of Anosov flows, Research announcemment, 1991.

[Su] D. Sullivan, Cycles for the study of foliated manifolds and complex manifolds, Invent. Math. 36 (1976), 225-255.

[Th1] W. Thurston, The geometry and topology of 3-manifolds, Princeton University Lecture Notes, 1982.

[Th2] W. Thurston, 3-dimensional manifolds, Kleinian groups and hyperbolic geometry, Bull. A.M.S. 6 (new series) (1982), 357-381.

[Ve] A. Verjovsky, Codimension one Anosov flows, Bol. Soc. Mat. Mex. 19 (1977), 49-77.

Sérgio R. Fenley

Washington University and

Princeton University

St. Louis, MO 63130, USA

(Received: March 13, 1997 ) 